\section{Database of proteinogenic amino acid reference spectra for Bismuth-cluster ToF-SIMS. I. Negative polarity}

\author{
Maciej Kawecki ${ }^{\text {a) }}$ \\ Laboratory of Nanoscale Materials Science, Empa, CH-8600 Dübendorf, Switzerland \\ and Department of Physics, University of Basel, CH-4056 Basel, Switzerland \\ Laetitia Bernard \\ Laboratory of Nanoscale Materials Science, Empa, CH-8600 Dübendorf, Switzerland
}

(Received 6 February 2018; accepted 1 May 2018; published 10 July 2018)

\begin{abstract}
The number of time-of-flight secondary ion mass spectrometry studies on biological tissues and cells strongly increased since the development of primary ion sources that allow not only elemental but also molecular analysis. Still, substantial fragmentation during ionic bombardment results in a large amount of peaks, rendering data analysis complex. Complete and trustable sets of reference spectra for the main biological building blocks such as proteins, sugars and lipids are required. Here, the authors provide a database of reference spectra for the 21 proteinogenic amino acids + glycine for bismuth instruments. Paper I of this two-part article series contains the negative polarity spectra and Paper II the positive polarity spectra. These reference spectra were obtained with the primary ion $\mathrm{Bi}_{3}{ }^{+}$and spectra for the primary ions $\mathrm{Bi}_{1}{ }^{+}$and $\mathrm{Bi}_{3}{ }^{++}$are contained in the supplementary material as ASCII files. Published by the AVS. https://doi.org/10.1116/1.5024987
\end{abstract}

Keywords: ToF-SIMS, amino acid, mass spectrometry, fragmentation

\begin{tabular}{l}
\hline Accession \#: 01426, 01427, 01428, \\
01429, 01430, 01431, 01432, \\
01433, 01434, 01435, 01436, \\
01437, 01438, 01439, 01440, \\
01441, 01442, 01443, 01444, \\
01445, 01446, 01447 \\
Technique: SIMS \\
Host Material: Silicon (100) wafer \\
Instrument: IONTOF TOF-SIMS.5 \\
Major Species in Spectra: C, H, N, \\
O, S \\
Minor Species in Spectra: Si, Cl \\
Published Spectra: 22 \\
Spectra in Electronic Record: 66 \\
Published Figures: 44 \\
Spectral Category: Reference
\end{tabular}

\section{INTRODUCTION}

Partial amino acid databases for time-of-flight secondary ion mass spectrometry (ToF-SIMS) reference mass spectra have been already conducted based on the now largely replaced monoatomic argon (Ref. 1) and caesium (Ref. 2) primary ion sources. The characteristic molecular fragmentation in ToF-SIMS measurements, however, depends on the primary ion impacting the surface. The current standard primary ion source is the bismuth/manganese liquid metal ion source. For bismuth and bismuth cluster primary ions, only limited lists attributing prominent peaks to specific amino acids can be found in the literature, e.g., Ref. 3. Here, we provide a database of original reference spectra for the 21 proteinogenic amino acids + glycine as well as the complete corresponding peak lists determining the fragmentation products. Fragments which uniquely represent specific amino acids are highlighted in Table 24. This Paper I of the database contains the negative polarity spectra. Positive polarity spectra are contained in Paper II (Ref. 4).

The amino acids were purchased through Sigma Aldrich (Article number: 09416, purity $>99.0 \%$ ). Each amino acid was dissolved in $\mathrm{H}_{2} \mathrm{O}$ (Sigma Aldrich, Article number: 270733) and $0.1 \mathrm{M}$ solutions were so prepared. Host silicon wafers of $1 \times 1 \mathrm{~cm}$ size were first washed by submersion in subsequent ultrasound baths of $2 \times 15 \mathrm{~min}$ in acetone and $1 \times 15$ minutes in ethanol, and then plasma-treated for a duration of $10 \mathrm{~min}$ to render the surface hydrophilic. The $0.1 \mathrm{M}$ amino acid solutions were subsequently drop-deposited inside a laminar flow chamber on separate silicon wafers. It was made sure that each deposited droplet is spread over the entire wafer surface before leaving the wafers in the laminar flow chamber for drying.

The spectra were obtained on a ToF-SIMS.5 instrument (IONTOF) equipped with a $25 \mathrm{keV}$ bismuth-manganese liquid metal

\footnotetext{
a)Electronic mail: maciej.kawecki@empa.ch
}

ion gun. The instrument was operated in high current bunched mode. The primary ion dose was kept below the static limit $\left(10^{12}\right.$ ions $/ \mathrm{cm}^{2}$ ) for each spectrum and a low-energy electron flood gun was used to compensate charging effects. The raster size was $200 \times 200 \mu \mathrm{m}^{2}$ with a raster resolution of $128 \times 128$, a cycle time of $100 \mu \mathrm{s}$, and primary ion currents of $1.54 \mathrm{pA}$ for $\mathrm{Bi}_{1}{ }^{+}$, 0.37- $0.4 \mathrm{pA}$ for $\mathrm{Bi}_{3}{ }^{+}$, and $0.44 \mathrm{pA}$ for $\mathrm{Bi}_{3}{ }^{++}$. Fifty scans were acquired per single mass spectrum. All presented spectra are normalized to their respective total ion count. Note that variations in mass resolution among the reference spectra (see Fig. 23) are due to crystallization geometry differences between the amino acids.

Figures and tables presented contain only attributions for the most prominent peaks. Complete peak-lists attributing fragmentation products to 50-100 main peaks for each amino acid are included in the attachment. In the figures, the full molecule is referred to with "M." Polymerization products are marked with "p.p.," peaks originating from the substrate with "s.," and contaminants with "c." Major peaks where a unique attribution was not possible, e.g., due to an overlap beyond mass resolution of two potential fragmentation products, are marked with "n.a." standing for not (uniquely) attributed. In the label of the supplementary material files, the Spectrum ID\# uniquely identifies each amino acid. Mass spectra acquired using $\mathrm{Bi}_{3}{ }^{+}, \mathrm{Bi}_{1}{ }^{+}$, and $\mathrm{Bi}_{3}{ }^{++}$primary ions are further identified by the file-suffix " 01, , " 02 ," and " 03 ," respectively, and peak lists in ASCII format by the file-suffix " 04 ."

SPECIMEN DESCRIPTION (ACCESSION \# 01426, 01427, 01428, 01429, 01430, 01431, 01432, 01433, 01434, 01435, 01436, 01437, 01438, 01439, 01440, 01441, 01442, 01443, 01444, 01445, 01446, 01447)

Host Material: Silicon (100) wafer 


\section{CAS Registry \#: 7440-21-3}

Host Material Characteristics: Homogeneous; solid; single crystal; semiconductor

Chemical Name: L-alanine (01426), L-arginine monohydrochloride (01427), L-asparagine (01428), L-asparatic acid (01429), Lcysteine (01430), L-cystine (01431), L-glutamic acid (01432), L-glutamine (01433), glycine (01434), L-histidine monohydrochloride (01435), L-4-hydroxiproline (01436), L-isoleucine (01437), L-leucine (01438), L-lysine monohydrochloride (01439), L-methionine (01440), L-phenylalanine (01441), Lproline (01442), L-serine (01443), L-threonine (01444), L-tryptophan (01445), L-tyrosine (01446), L-valine (01447)

Source: Sigma Aldrich

Host Composition: $\mathrm{Si}$ and $\mathrm{SiO}_{2}$

Form: Crystalline powder dissolved in $\mathrm{DI} \mathrm{H}_{2} \mathrm{O}$ and subsequently drop-deposited on the host wafer

Lot Number: L-alanine: BCBS2461V, L-arginine monohydrochloride: 1361811V, L-asparagine: 1371738V, L-asparatic acid: BCBR6815V, L-cysteine: BCBR4500V, L-cystine: BCBS1903V, L-glutamic acid: $1423805 \mathrm{~V}$, L-glutamine: BCBR7569V, glycine: 1119375V, L-histidine monohydrochloride: BCBS1876V, L-4-hydroxiproline: BCBL2666V, L-isoleucine: 1423806V, L-leucine: BCBBQ9986V, L-lysine monohydrochloride: BCBN9886V, L-methionine: $1423807 \mathrm{~V}, \mathrm{~L}-$ phenylalanine: BCBQ1493V, L-proline: BCBP4505V, L-serine: 1336081V, L-threonine: BCBD4901V, L-tryptophan: BCBR0168V, L-tyrosine: BCBR7417V, L-valine: BCBM0163V

Structure: L-alanine: $\mathrm{C}_{3} \mathrm{H}_{7} \mathrm{NO}_{2}$, L-arginine monohydrochloride: $\mathrm{C}_{6} \mathrm{H}_{14} \mathrm{~N}_{4} \mathrm{O}_{2} \cdot \mathrm{HCl}$, L-asparagine: $\mathrm{C}_{4} \mathrm{H}_{8} \mathrm{~N}_{2} \mathrm{O}_{3}$, L-asparatic acid: $\mathrm{C}_{4} \mathrm{H}_{7} \mathrm{NO}_{4}$, L-cysteine: $\mathrm{C}_{3} \mathrm{H}_{7} \mathrm{NO}_{2} \mathrm{~S}$, L-cystine: $\mathrm{C}_{6} \mathrm{H}_{12} \mathrm{~N}_{2} \mathrm{O}_{4} \mathrm{~S}_{2}$, L-glutamic acid: $\mathrm{C}_{5} \mathrm{H}_{9} \mathrm{NO}_{4}$, L-glutamine: $\mathrm{C}_{5} \mathrm{H}_{10} \mathrm{~N}_{2} \mathrm{O}_{3}$, glycine: $\mathrm{C}_{2} \mathrm{H}_{5} \mathrm{NO}_{2}$, L-histidine monohydrochloride: $\mathrm{C}_{6} \mathrm{H}_{9} \mathrm{~N}_{3} \mathrm{O}_{2} \cdot \mathrm{HCl}$, L-4-hydroxiproline: $\mathrm{C}_{5} \mathrm{H}_{9} \mathrm{NO}_{3}$, L-isoleucine: $\mathrm{C}_{6} \mathrm{H}_{13} \mathrm{NO}_{2}$, L-leucine: $\mathrm{C}_{6} \mathrm{H}_{13} \mathrm{NO}_{2}$, L-lysine monohydrochloride: $\mathrm{C}_{6} \mathrm{H}_{14} \mathrm{~N}_{2} \mathrm{O}_{2} \cdot \mathrm{HCl}$, L-methionine: $\mathrm{C}_{5} \mathrm{H}_{11} \mathrm{NO}_{2} \mathrm{~S}$, L-phenylalanine: $\mathrm{C}_{9} \mathrm{H}_{11} \mathrm{NO}_{2}$, L-proline: $\mathrm{C}_{5} \mathrm{H}_{9} \mathrm{NO}_{2}$, L-serine: $\mathrm{C}_{3} \mathrm{H}_{7} \mathrm{NO}_{3}$, L-threonine: $\mathrm{C}_{4} \mathrm{H}_{9} \mathrm{NO}_{3}$, Ltryptophan: $\mathrm{C}_{11} \mathrm{H}_{12} \mathrm{~N}_{2} \mathrm{O}_{2}$, L-tyrosine: $\mathrm{C}_{9} \mathrm{H}_{11} \mathrm{NO}_{3}$, L-valine: $\mathrm{C}_{5} \mathrm{H}_{11} \mathrm{NO}_{2}$

History and Significance: The proteinogenic amino acids are the building blocks of all proteins in the known life forms. Complete spectra databases of organic molecules facilitate the interpretation of ToF-SIMS data from biological specimen. However, it should be kept in mind that in biological specimens amino acids are incorporated into larger molecules (proteins, peptidoglycans, etc.), which affects the fragmentation patterns.

As Received Condition: Samples received in powder form

Analyzed Region: Regions of $200 \times 200 \mu \mathrm{m}^{2}$ on maximally homogeneous specimen sites

Ex Situ Preparation/Mounting: 0.1 M amino acid solutions were prepared in $\mathrm{H}_{2} \mathrm{O}$ and drop-deposited on separate $1 \times 1 \mathrm{~cm}$ large plasma-treated $\mathrm{Si}$-wafers in a laminar flow chamber.

In Situ Preparation: None

Charge Control: Low energy electrons

Temp. During Analysis: $300 \mathrm{~K}$
Pressure During Analysis: $5.6 \times 10^{-8} \mathrm{~Pa}$

Preanalysis Beam Exposure: None

\section{INSTRUMENT CONFIGURATION}

Manufacturer and Model: IONTOF TOF-SIMS.5

Analyzer Type: Time-of-flight

Detector Type: Microchannel plate

Experiment Type: Mass spectra

Oxygen Flood Source: None

Other Flood Source: Low energy electrons

Energy Acceptance Window: $20 \mathrm{eV}$

Postacceleration Voltage: $10000 \mathrm{eV}$

Sample Bias: $0 \mathrm{eV}$

Specimen Normal-to-analyzer (囚e): $0^{\circ}$

\section{- Ion Sources}

\section{Ion Source 1 of 1}

Purpose of this Ion Source: Analysis beam

Ion Source Manufacturer: IONTOF (Münster, Germany)

Ion Source Model: $25 \mathrm{keV} \mathrm{Bi/Mn} \mathrm{cluster} \mathrm{source}$

Beam Mass Filter: Electrodynamic mass filter

Beam Species and Charge State: $\mathrm{Bi}_{3}{ }^{+}$

Beam Gating Used: None

Beam Voltage: $25000 \mathrm{eV}$

Net Beam Voltage (impact voltage): $25000 \mathrm{eV}$

Ion Pulse Width: $29.3 \mathrm{~ns}$

Ion Pulse Rate: $10 \mathrm{kHz}$

Pulsed Beam Current: 0.00037-0.0004 nA

Current Measurement Method: Faraday cup

Beam Raster Size: $200 \times 200 \mu \mathrm{m}^{2}$

Beam Incident Angle: $45^{\circ}$

Source-to-Analyzer Angle: $45^{\circ}$

\section{ACKNOWLEDGMENTS}

The authors are thankful for the funding received from the Swiss National Science Foundation through the Grant No. CR23I2162828 .

\section{REFERENCES}

1. Preinstalled database on IONTOF GmbH instruments.

2. N. T. Samuel, M. S. Wagner, K. D. Dornfeld, and D. G. Castner, Surf. Sci. Spectra 8, 163 (2001).

3. M. Urbini, V. Piteto, F. de Notaristefani, F. Scaldaferri, A. Gasbarrini, and L. Tortora, Anal. Bioanal. Chem. 409, 6097 (2017).

4. M. Kawecki and L. Bernard, Surf. Sci. Spectra 25, 015002 (2018). 


\begin{tabular}{lccc}
\hline \multicolumn{3}{c}{ ALANINE } \\
\hline Spectrum ID \# & Mass (Da) & Species & Peak Assignment \\
\hline $\mathbf{0 1 4 2 6}$ & 71.0139 & $\mathrm{C}_{3} \mathrm{H}_{3} \mathrm{O}_{2}^{-}$ & Alanine \\
$\ldots$ & 72.0091 & $\mathrm{C}_{2} \mathrm{H}_{2} \mathrm{NO}_{2}^{-}$ & $\ldots$ \\
$\ldots$ & 86.0248 & $\mathrm{C}_{3} \mathrm{H}_{4} \mathrm{NO}_{2}^{-}$ & $\ldots$ \\
$\ldots$ & 88.0404 & $\mathrm{C}_{3} \mathrm{H}_{6} \mathrm{NO}_{2}^{-}$ & $\ldots$ \\
$\ldots$ & 89.0482 & $\mathrm{C}_{3} \mathrm{H}_{7} \mathrm{NO}_{2}^{-}$ & $\ldots$ \\
\hline
\end{tabular}

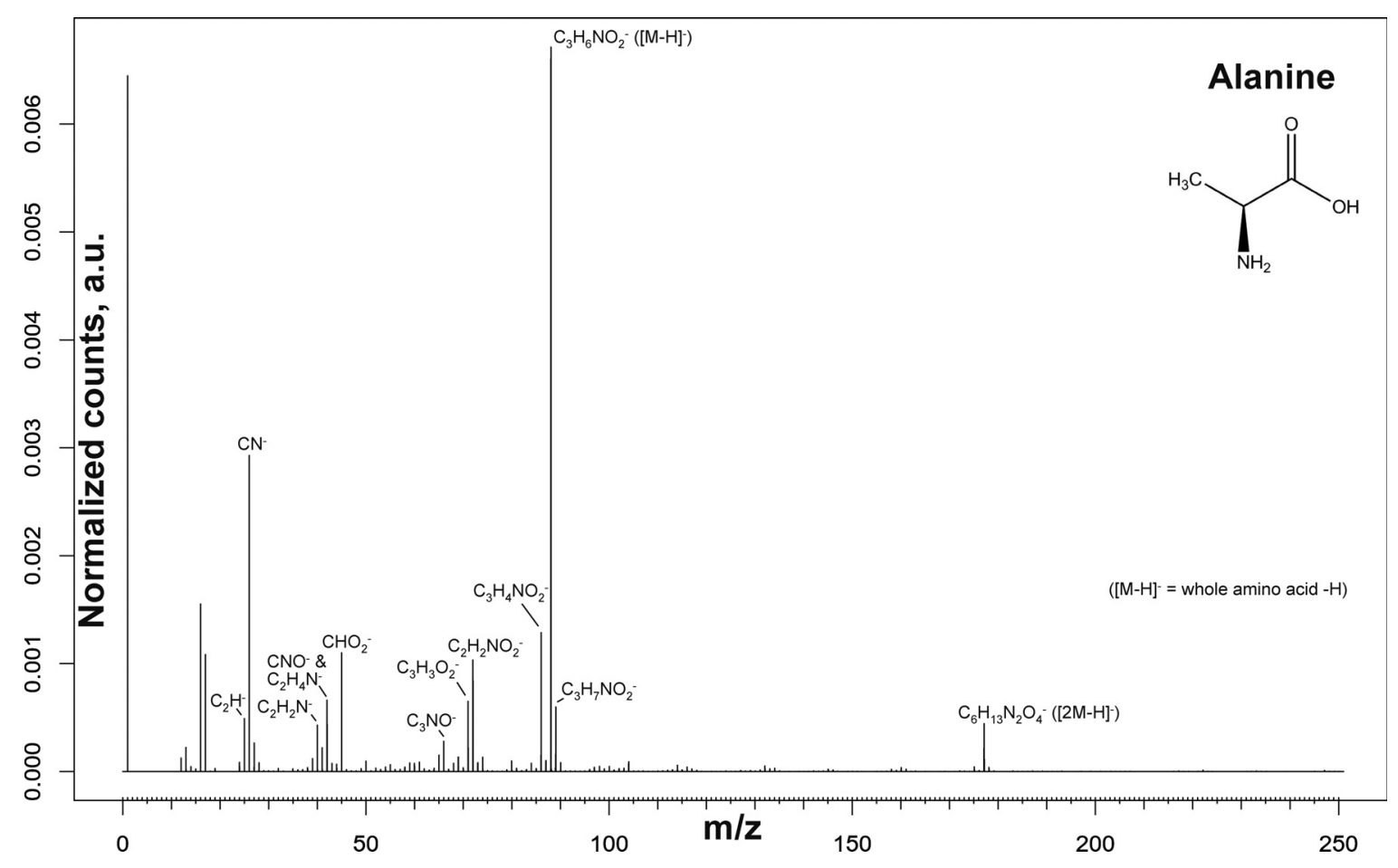

\begin{tabular}{rc}
\hline Accession \# & 01426-01, 01426-02, 01426-03, 01426-04 \\
\hline Host Material & Silicon wafer \\
Technique & SIMS \\
Secondary Source Polarity & Negative \\
Mass Range Minimum & $0 \mathrm{Da}$ \\
Mass Range Maximum & $250 \mathrm{Da}$ \\
Species Used for Mass Calibration & $\mathrm{CH}^{-}, \mathrm{OH}^{-}, \mathrm{C}_{2} \mathrm{H}^{-}, \mathrm{CH}_{2} \mathrm{~N}^{-}, \mathrm{C}_{3} \mathrm{H}_{4} \mathrm{NO}_{2}{ }^{-}$ \\
Primary lon Dose & $5.11 \times 10^{11} \mathrm{~cm}^{-2}$ \\
Primary Species & $\mathrm{Bi}_{3}^{+}$ \\
Primary lon Pulse Width & $29.3 \mathrm{~ns}^{+}$ \\
Pulsed Beam Current & $0.0004 \mathrm{nA}^{2}$ \\
Beam Raster Size & $200 \times 200 \mu \mathrm{m}^{2}$ \\
\hline
\end{tabular}




\section{ARGININE (HYDROCHLORIDE)}

\begin{tabular}{lccc}
\hline Spectrum ID \# & Mass (Da) & Species & Peak Assignment \\
\hline $\mathbf{0 1 4 2 7}$ & 41.0145 & $\mathrm{CHN}_{2}^{-}$ & Arginine \\
$\ldots$ & 58.0411 & $\mathrm{CH}_{4} \mathrm{~N}_{3}^{-}$ & $\ldots$ \\
$\ldots$ & 131.0826 & $\mathrm{C}_{5} \mathrm{H}_{11} \mathrm{~N}_{2} \mathrm{O}_{2}^{-}$ & $\ldots$ \\
$\ldots$ & 156.0779 & $\mathrm{C}_{6} \mathrm{H}_{10} \mathrm{~N}_{3} \mathrm{O}_{2}^{-}$ & $\ldots$ \\
$\ldots$ & 158.0935 & $\mathrm{C}_{6} \mathrm{H}_{12} \mathrm{~N}_{3} \mathrm{O}_{2}^{-}$ & $\ldots$ \\
$\ldots$ & 173.1044 & $\mathrm{C}_{6} \mathrm{H}_{13} \mathrm{~N}_{4} \mathrm{O}_{2}^{-}$ & $\ldots$ \\
\hline
\end{tabular}

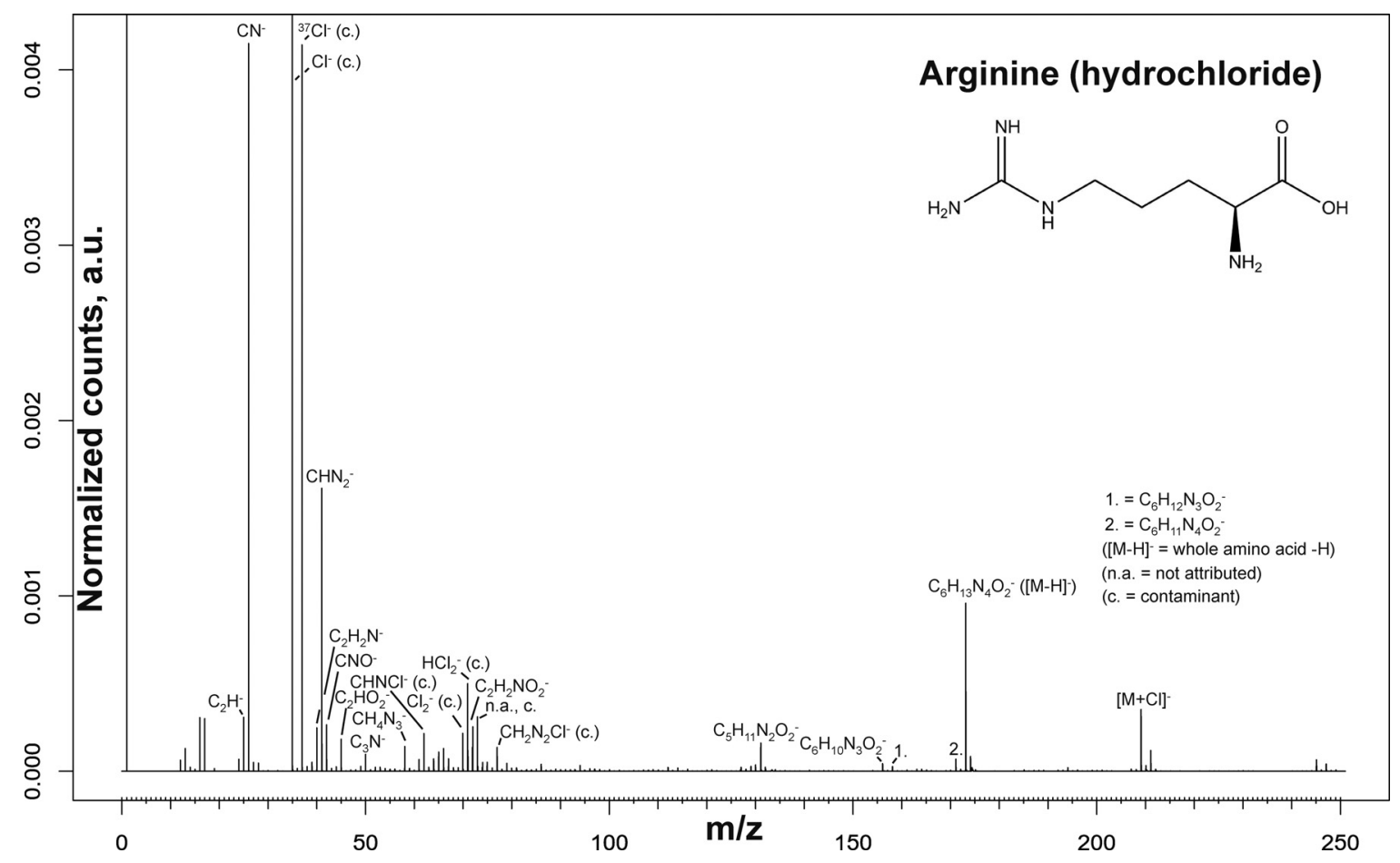

Accession \#

01427-01, 01427-02, 01427-03, 01427-04

Host Material

- Technique

Secondary Source Polarity

Mass Range Minimum

Mass Range Maximum

Species Used for Mass Calibration

Primary lon Dose

Primary Species

Primary lon Pulse Width

Pulsed Beam Current

Beam Raster Size
Silicon wafer

SIMS

Negative

$0 \mathrm{Da}$

$250 \mathrm{Da}$

$\mathrm{CH}^{-}, \mathrm{OH}^{-}, \mathrm{C}_{2} \mathrm{H}^{-}, \mathrm{CH}_{2} \mathrm{~N}^{-}, \mathrm{C}_{6} \mathrm{H}_{14} \mathrm{~N}_{4} \mathrm{O}_{2}{ }^{-}$

$4.73 \times 10^{11} \mathrm{~cm}^{-2}$

$\mathrm{Bi}_{3}{ }^{+}$

$29.3 \mathrm{~ns}$

$0.00037 \mathrm{nA}$

$200 \times 200 \mu \mathrm{m}^{2}$ 


\begin{tabular}{lccc}
\hline \multicolumn{3}{c}{ ASPARAGINE } \\
\hline Spectrum ID \# & Mass (Da) & Species & Peak Assignment \\
\hline $\mathbf{0 1 4 2 8}$ & 41.9985 & $\mathrm{CNO}^{-}$ & Asparagine \\
$\ldots$ & 72.0091 & $\mathrm{C}_{2} \mathrm{H}_{2} \mathrm{NO}_{2}^{-}$ & $\ldots$ \\
$\ldots$ & 113.0357 & $\mathrm{C}_{4} \mathrm{H}_{5} \mathrm{~N}_{2} \mathrm{O}_{2}^{-}$ & $\cdots$ \\
$\ldots$ & 114.0197 & $\mathrm{C}_{4} \mathrm{H}_{4} \mathrm{NO}_{3}{ }^{-}$ & $\ldots$ \\
$\ldots$ & 131.0462 & $\mathrm{C}_{4} \mathrm{H}_{7} \mathrm{~N}_{2} \mathrm{O}_{3}{ }^{-}$ & $\ldots$ \\
\hline
\end{tabular}

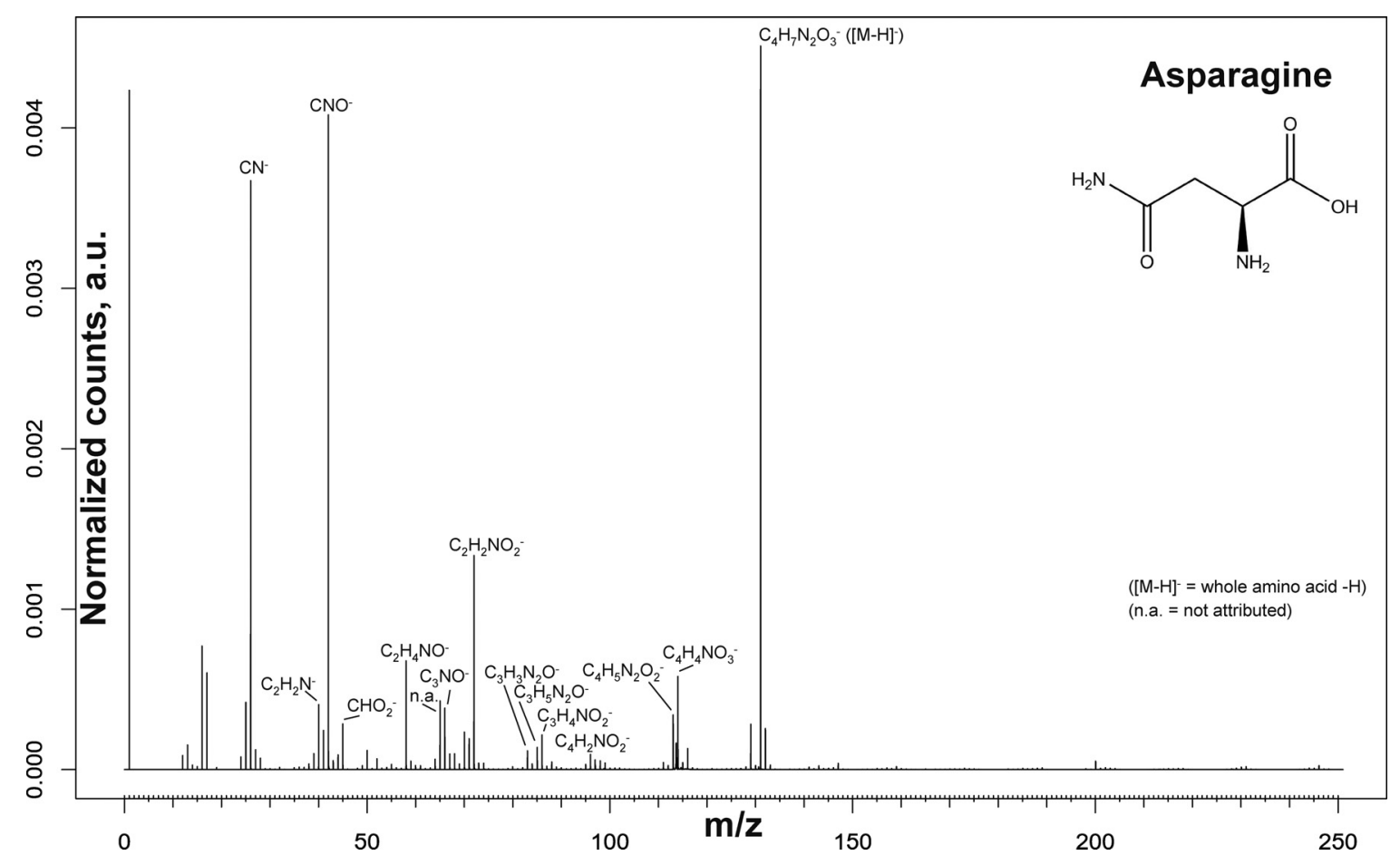

Accession \#

- Host Material

- Technique

Secondary Source Polarity

Mass Range Minimum

Mass Range Maximum

Species Used for Mass Calibration

Primary lon Dose

Primary Species

Primary lon Pulse Width

Pulsed Beam Current

Beam Raster Size
01428-01, 01428-02, 01428-03, 01428-04

Silicon wafer

SIMS

Negative

$0 \mathrm{Da}$

$250 \mathrm{Da}$

$\mathrm{CH}^{-}, \mathrm{OH}^{-}, \mathrm{C}_{2} \mathrm{H}^{-}, \mathrm{CH}_{2} \mathrm{~N}^{-}, \mathrm{C}_{4} \mathrm{H}_{5} \mathrm{~N}_{2} \mathrm{O}_{3}^{-}$

$4.73 \times 10^{11} \mathrm{~cm}^{-2}$

$\mathrm{Bi}_{3}{ }^{+}$

$29.3 \mathrm{~ns}$

$0.00037 \mathrm{nA}$

$200 \times 200 \mu \mathrm{m}^{2}$ 


\begin{tabular}{lccc}
\hline \multicolumn{3}{c}{ ASPARATIC ACID } \\
\hline Spectrum ID \# & Mass (Da) & Species & Peak Assignment \\
\hline $\mathbf{0 1 4 2 9}$ & 98.0248 & $\mathrm{C}_{4} \mathrm{H}_{4} \mathrm{NO}_{2}{ }^{-}$ & Asparatic acid \\
$\ldots$ & 88.0404 & $\mathrm{C}_{3} \mathrm{H}_{6} \mathrm{NO}_{2}{ }^{-}$ & $\ldots$ \\
$\ldots$ & 115.0037 & $\mathrm{C}_{4} \mathrm{H}_{3} \mathrm{O}_{4}^{-}$ & $\ldots$ \\
$\ldots$ & 117.0193 & $\mathrm{C}_{4} \mathrm{H}_{5} \mathrm{O}_{4}^{-}$ & $\ldots$ \\
$\ldots$ & 132.0302 & $\mathrm{C}_{4} \mathrm{H}_{6} \mathrm{NO}_{4}^{-}$ & $\ldots$ \\
\hline
\end{tabular}

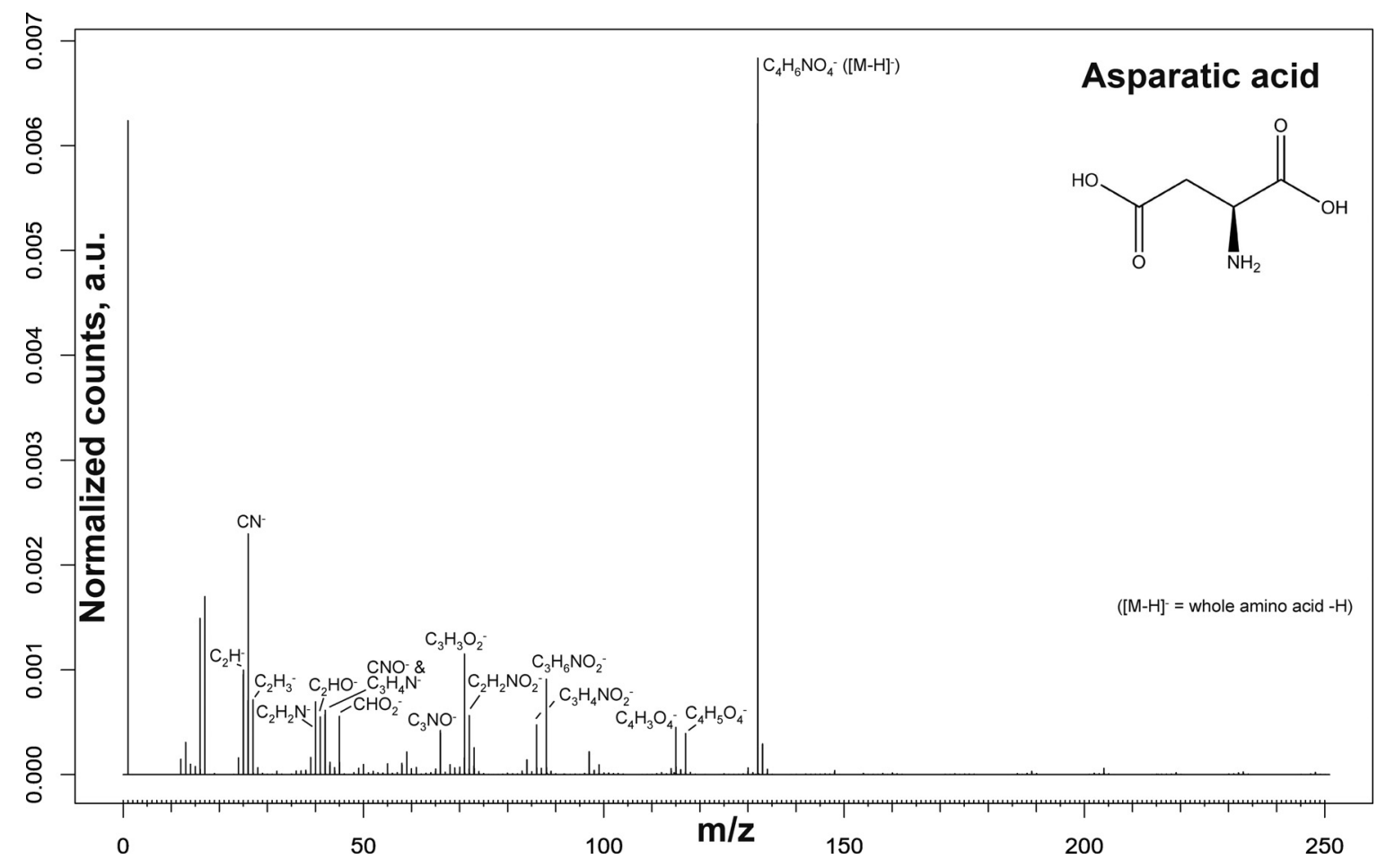

Accession \#

Host Material

- Technique

Secondary Source Polarity

Mass Range Minimum

Mass Range Maximum

Species Used for Mass Calibration

Primary lon Dose

Primary Species

Primary lon Pulse Width

Pulsed Beam Current

Beam Raster Size
01429-01, 01429-02, 01429-03, 01429-04

Silicon wafer

SIMS

Negative

$0 \mathrm{Da}$

$250 \mathrm{Da}$

$\mathrm{CH}^{-}, \mathrm{OH}^{-}, \mathrm{C}_{2} \mathrm{H}^{-}, \mathrm{CH}_{2} \mathrm{~N}^{-}, \mathrm{C}_{4} \mathrm{H}_{7} \mathrm{NO}_{4}^{-}$

$4.73 \times 10^{11} \mathrm{~cm}^{-2}$

$\mathrm{Bi}_{3}{ }^{+}$

$29.3 \mathrm{~ns}$

$0.00037 \mathrm{nA}$

$200 \times 200 \mu \mathrm{m}^{2}$ 


\begin{tabular}{lccc}
\hline & & CYSTEINE & \\
\hline Spectrum ID \# & Mass (Da) & Species & Peak Assignment \\
\hline $\mathbf{0 1 4 3 0}$ & 31.9726 & $\mathrm{~S}^{-}$ & Cysteine \\
$\ldots$ & 32.9804 & $\mathrm{HS}^{-}$ & $\ldots$ \\
$\ldots$ & 56.9804 & $\mathrm{C}_{2} \mathrm{HS}^{-}$ & $\ldots$ \\
$\ldots$ & 57.9757 & $\mathrm{CNS}^{-}$ & $\ldots$ \\
$\ldots$ & 72.0091 & $\mathrm{C}_{2} \mathrm{H}_{2} \mathrm{NO}^{-}$ & $\ldots$ \\
$\ldots$ & 74.0070 & $\mathrm{C}_{2} \mathrm{H}_{4} \mathrm{NS}^{-}$ & $\ldots$ \\
$\ldots$ & 86.0248 & $\mathrm{C}_{3} \mathrm{H}_{4} \mathrm{NO}_{2}$ & $\ldots$ \\
$\ldots$ & 102.9859 & $\mathrm{C}_{3} \mathrm{H}_{3} \mathrm{O}_{2} \mathrm{~S}^{-}$ & $\ldots$ \\
$\ldots$ & 120.0125 & $\mathrm{C}_{3} \mathrm{H}_{6} \mathrm{NO}_{2} \mathrm{~S}^{-}$ & $\ldots$ \\
\hline
\end{tabular}

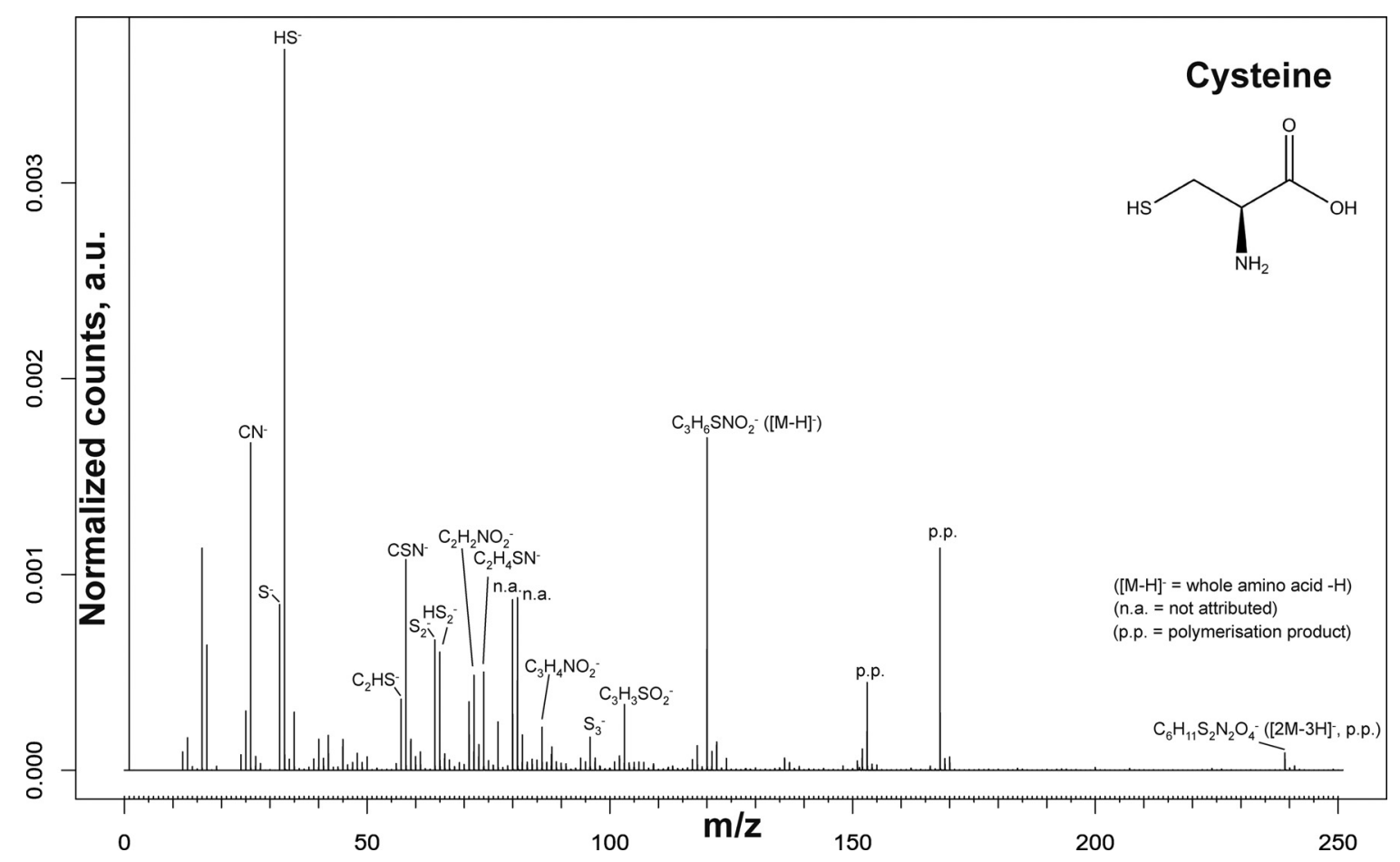

Accession \#

Host Material

- Technique

Secondary Source Polarity

Mass Range Minimum

Mass Range Maximum

Species Used for Mass Calibration

Primary Ion Dose

Primary Species

Primary lon Pulse Width

Pulsed Beam Current

Beam Raster Size
01430-01, 01430-02, 01430-03, 01430-04

Silicon wafer

SIMS

Negative

$0 \mathrm{Da}$

$250 \mathrm{Da}$

$\mathrm{CH}^{-}, \mathrm{OH}^{-}, \mathrm{C}_{2} \mathrm{H}^{-}, \mathrm{CH}_{2} \mathrm{~N}^{-}, \mathrm{C}_{3} \mathrm{H}_{7} \mathrm{SNO}_{2}^{-}$

$4.73 \times 10^{11} \mathrm{~cm}^{-2}$

$\mathrm{Bi}_{3}{ }^{+}$

$29.3 \mathrm{~ns}$

$0.00037 \mathrm{nA}$

$200 \times 200 \mu \mathrm{m}^{2}$ 


\begin{tabular}{lccc}
\hline & \multicolumn{3}{c}{ CYSTINE } \\
\hline Spectrum ID \# & Mass (Da) & Species & Peak Assignment \\
\hline $\mathbf{0 1 4 3 1}$ & 31.9726 & $\mathrm{~S}^{-}$ & Cystine \\
$\ldots$ & 32.9804 & $\mathrm{HS}^{-}$ & $\ldots$ \\
$\ldots$ & 63.9447 & $\mathrm{~S}_{2}{ }^{-}$ & $\ldots$ \\
$\ldots$ & 64.9525 & $\mathrm{HS}_{2}^{-}$ & $\ldots$ \\
$\ldots$ & 74.0070 & $\mathrm{C}_{2} \mathrm{H}_{4} \mathrm{NS}^{-}$ & $\ldots$ \\
$\ldots$ & 104.0176 & $\mathrm{C}_{3} \mathrm{H}_{6} \mathrm{NOS}^{-}$ & $\ldots$ \\
$\ldots$ & 120.0125 & $\mathrm{C}_{3} \mathrm{H}_{6} \mathrm{NO}_{2} \mathrm{~S}^{-}$ & $\ldots$ \\
$\ldots$ & 151.9845 & $\mathrm{C}_{3} \mathrm{H}_{6} \mathrm{NO}_{2} \mathrm{~S}_{2}^{-}$ & $\ldots$ \\
$\ldots$ & 239.0166 & $\mathrm{C}_{6} \mathrm{H}_{11} \mathrm{~N}_{2} \mathrm{O}_{4} \mathrm{~S}_{2}^{-}$ & $\ldots$ \\
\hline
\end{tabular}

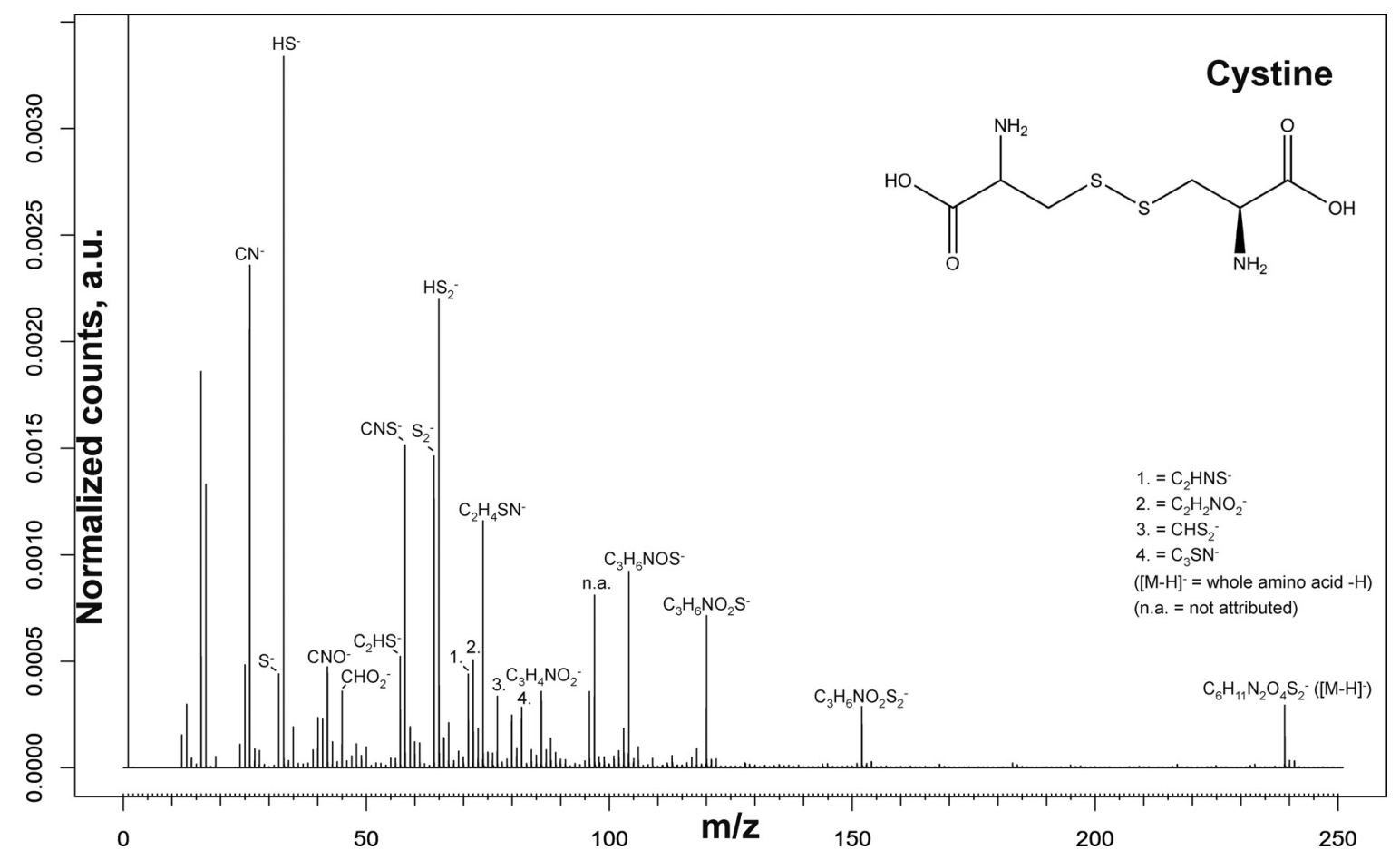

\begin{tabular}{|c|c|}
\hline Accession \# & 01431-01, 01431-02, 01431-03, 01431-04 \\
\hline - Host Material & Silicon wafer \\
\hline Technique & SIMS \\
\hline Secondary Source Polarity & Negative \\
\hline Mass Range Minimum & $0 \mathrm{Da}$ \\
\hline Mass Range Maximum & $250 \mathrm{Da}$ \\
\hline Species Used for Mass Calibration & $\mathrm{CH}^{-}, \mathrm{OH}^{-}, \mathrm{C}_{2} \mathrm{H}^{-}, \mathrm{CH}_{2} \mathrm{~N}^{-}, \mathrm{C}_{6} \mathrm{H}_{11} \mathrm{~S}_{2} \mathrm{~N}_{2} \mathrm{O}_{4}^{-}$ \\
\hline Primary Ion Dose & $4.73 \times 10^{11} \mathrm{~cm}^{-2}$ \\
\hline Primary Species & $\mathrm{Bi}_{3}^{+}$ \\
\hline Primary Ion Pulse Width & $29.3 \mathrm{~ns}$ \\
\hline Pulsed Beam Current & $0.00037 \mathrm{nA}$ \\
\hline Beam Raster Size & $200 \times 200 \mu \mathrm{m}^{2}$ \\
\hline Comment & $\begin{array}{c}\text { Region of interest (ROI), defined by a minimum threshold of the } \mathrm{HS}_{2}{ }^{-} \text {signal, } \\
\text { was applied to eliminate the substrate signal. }\end{array}$ \\
\hline
\end{tabular}




\begin{tabular}{lccc}
\hline & \multicolumn{2}{c}{ GLUTAMIC ACID } & \\
\hline Spectrum ID \# & Mass (Da) & Species & Peak Assignment \\
\hline $\mathbf{0 1 4 3 2}$ & 59.0139 & $\mathrm{C}_{2} \mathrm{H}_{3} \mathrm{O}_{2}^{-}$ & Glutamic acid \\
$\ldots$ & 71.0139 & $\mathrm{C}_{3} \mathrm{H}_{3} \mathrm{O}_{2}^{-}$ & $\ldots$ \\
$\ldots$ & 72.0091 & $\mathrm{C}_{2} \mathrm{H}_{2} \mathrm{NO}_{2}^{-}$ & $\ldots$ \\
$\ldots$ & 74.0248 & $\mathrm{C}_{2} \mathrm{H}_{4} \mathrm{NO}_{2}^{-}$ & $\ldots$ \\
$\ldots$ & 85.0295 & $\mathrm{C}_{4} \mathrm{H}_{5} \mathrm{O}_{2}^{-}$ & $\ldots$ \\
$\ldots$ & 100.0404 & $\mathrm{C}_{4} \mathrm{H}_{6} \mathrm{NO}_{2}^{-}$ & $\ldots$ \\
$\ldots$ & 102.0560 & $\mathrm{C}_{4} \mathrm{H}_{8} \mathrm{NO}_{2}^{-}$ & $\ldots$ \\
$\ldots$ & 128.0353 & $\mathrm{C}_{5} \mathrm{H}_{6} \mathrm{NO}_{3}^{-}$ & $\ldots$ \\
$\ldots$ & 131.0350 & $\mathrm{C}_{5} \mathrm{H}_{7} \mathrm{O}_{4}^{-}$ & $\ldots$ \\
\hline
\end{tabular}

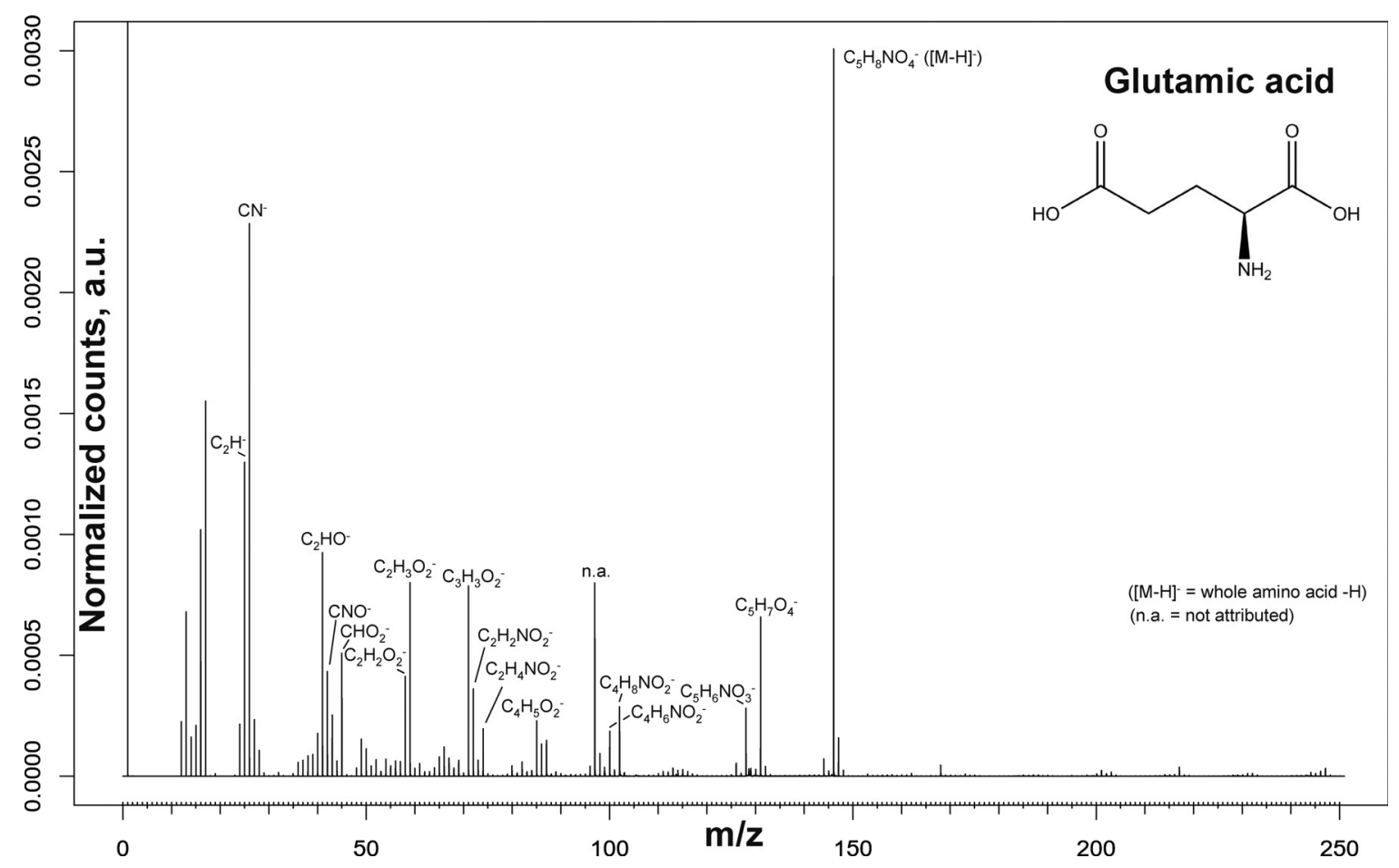

Accession \#

Host Material

- Technique

Secondary Source Polarity

Mass Range Minimum

Mass Range Maximum

Species Used for Mass Calibration

Primary Ion Dose

Primary Species

Primary Ion Pulse Width

Pulsed Beam Current

Beam Raster Size
01432-01, 01432-02, 01432-03, 01432-04

Silicon wafer

SIMS

Negative

$0 \mathrm{Da}$

$250 \mathrm{Da}$

$\mathrm{CH}^{-}, \mathrm{OH}^{-}, \mathrm{C}_{2} \mathrm{H}^{-}, \mathrm{CH}_{2} \mathrm{~N}^{-}, \mathrm{C}_{5} \mathrm{H}_{9} \mathrm{NO}_{4}{ }^{-}$

$4.73 \times 10^{11} \mathrm{~cm}^{-2}$

$\mathrm{Bi}_{3}^{+}$

$29.3 \mathrm{~ns}$

$0.00037 \mathrm{nA}$

$200 \times 200 \mu \mathrm{m}^{2}$ 


\begin{tabular}{lccc}
\hline \multicolumn{3}{c}{ GLUTAMINE } & \\
\hline Spectrum ID \# & Mass (Da) & Species & Peak Assignment \\
\hline $\mathbf{0 1 4 3 3}$ & 41.9985 & $\mathrm{CNO}^{-}$ & Glutamine \\
$\ldots$ & 58.0298 & $\mathrm{C}_{2} \mathrm{H}_{4} \mathrm{NO}^{-}$ & $\ldots$ \\
$\ldots$ & 72.0091 & $\mathrm{C}_{2} \mathrm{H}_{2} \mathrm{NO}_{2}^{-}$ & $\ldots$ \\
$\ldots$ & 74.0248 & $\mathrm{C}_{2} \mathrm{H}_{4} \mathrm{NO}_{2}^{-}$ & $\ldots$ \\
$\ldots$ & 82.0298 & $\mathrm{C}_{4} \mathrm{H}_{4} \mathrm{NO}^{-}$ & $\ldots$ \\
$\ldots$ & 99.0564 & $\mathrm{C}_{4} \mathrm{H}_{7} \mathrm{~N}_{2} \mathrm{O}^{-}$ & $\ldots$ \\
$\ldots$ & 128.0353 & $\mathrm{C}_{5} \mathrm{H}_{6} \mathrm{NO}_{3}^{-}$ & $\ldots$ \\
$\ldots$ & 145.0619 & $\mathrm{C}_{5} \mathrm{H}_{9} \mathrm{~N}_{2} \mathrm{O}_{3}^{-}$ & $\ldots$ \\
\hline
\end{tabular}

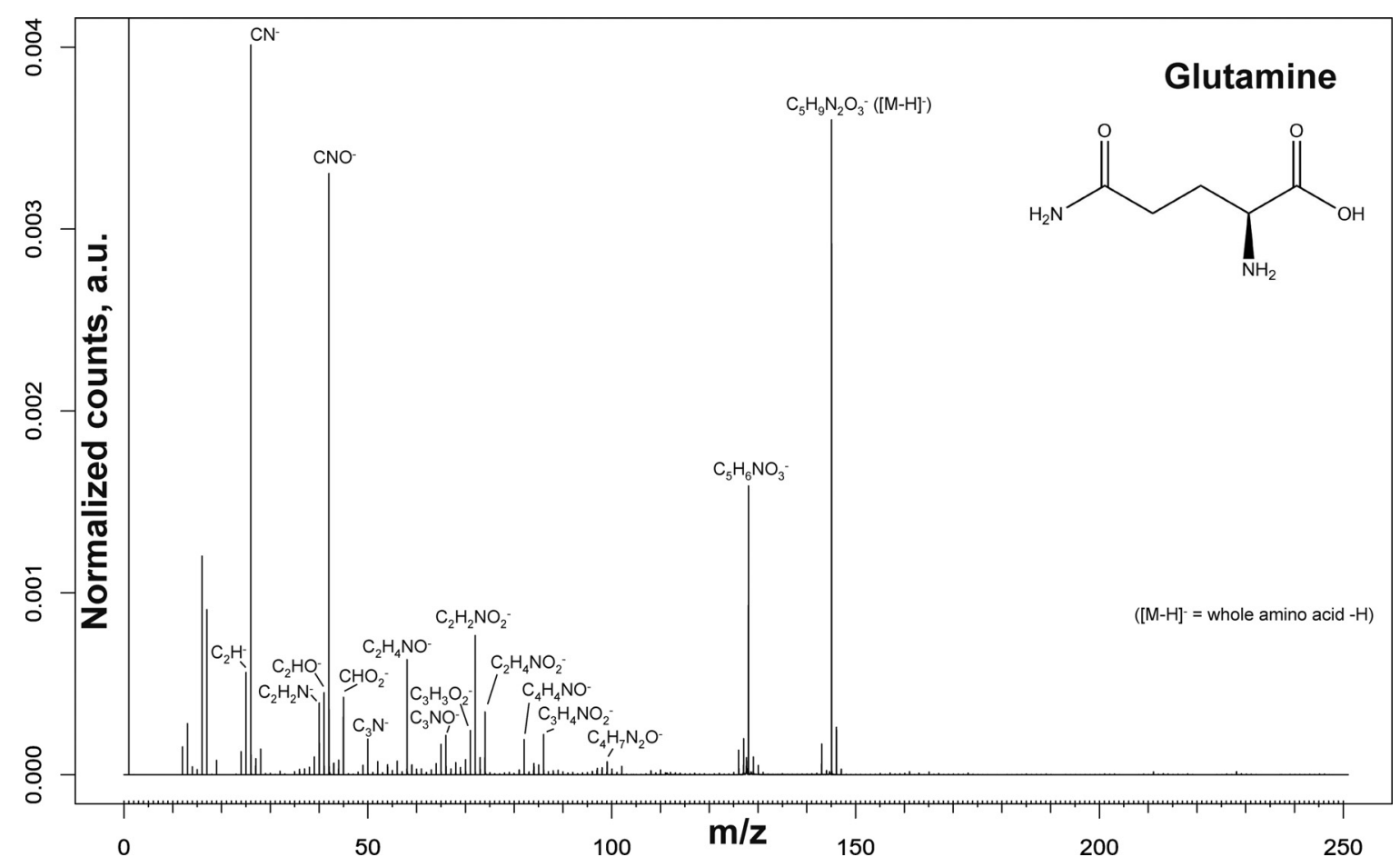

Accession \#

- Host Material

- Technique

Secondary Source Polarity

Mass Range Minimum

Mass Range Maximum

Species Used for Mass Calibration

Primary lon Dose

Primary Species

Primary lon Pulse Width

Pulsed Beam Current

Beam Raster Size
01433-01, 01433-02, 01433-03, 01433-04

Silicon wafer

SIMS

Negative

$0 \mathrm{Da}$

$250 \mathrm{Da}$

$\mathrm{CH}^{-}, \mathrm{OH}^{-}, \mathrm{C}_{2} \mathrm{H}^{-}, \mathrm{CH}_{2} \mathrm{~N}^{-}, \mathrm{C}_{5} \mathrm{H}_{7} \mathrm{~N}_{2} \mathrm{O}_{3}^{-}$

$4.73 \times 10^{11} \mathrm{~cm}^{-2}$

$\mathrm{Bi}_{3}{ }^{+}$

$29.3 \mathrm{~ns}$

$0.00037 \mathrm{nA}$

$200 \times 200 \mu \mathrm{m}^{2}$ 


\begin{tabular}{lccc}
\hline \multicolumn{3}{c}{ GLYCINE } \\
\hline Spectrum ID \# & Mass (Da) & Species & Peak Assignment \\
\hline $\mathbf{0 1 4 3 4}$ & 58.0060 & $\mathrm{C}_{2} \mathrm{H}_{2} \mathrm{O}_{2}^{-}$ & Glycine \\
$\ldots$ & 72.0091 & $\mathrm{C}_{2} \mathrm{H}_{2} \mathrm{NO}_{2}^{-}$ & $\ldots$ \\
$\ldots$ & 74.0248 & $\mathrm{C}_{2} \mathrm{H}_{4} \mathrm{NO}_{2}^{-}$ & $\ldots$ \\
\hline
\end{tabular}

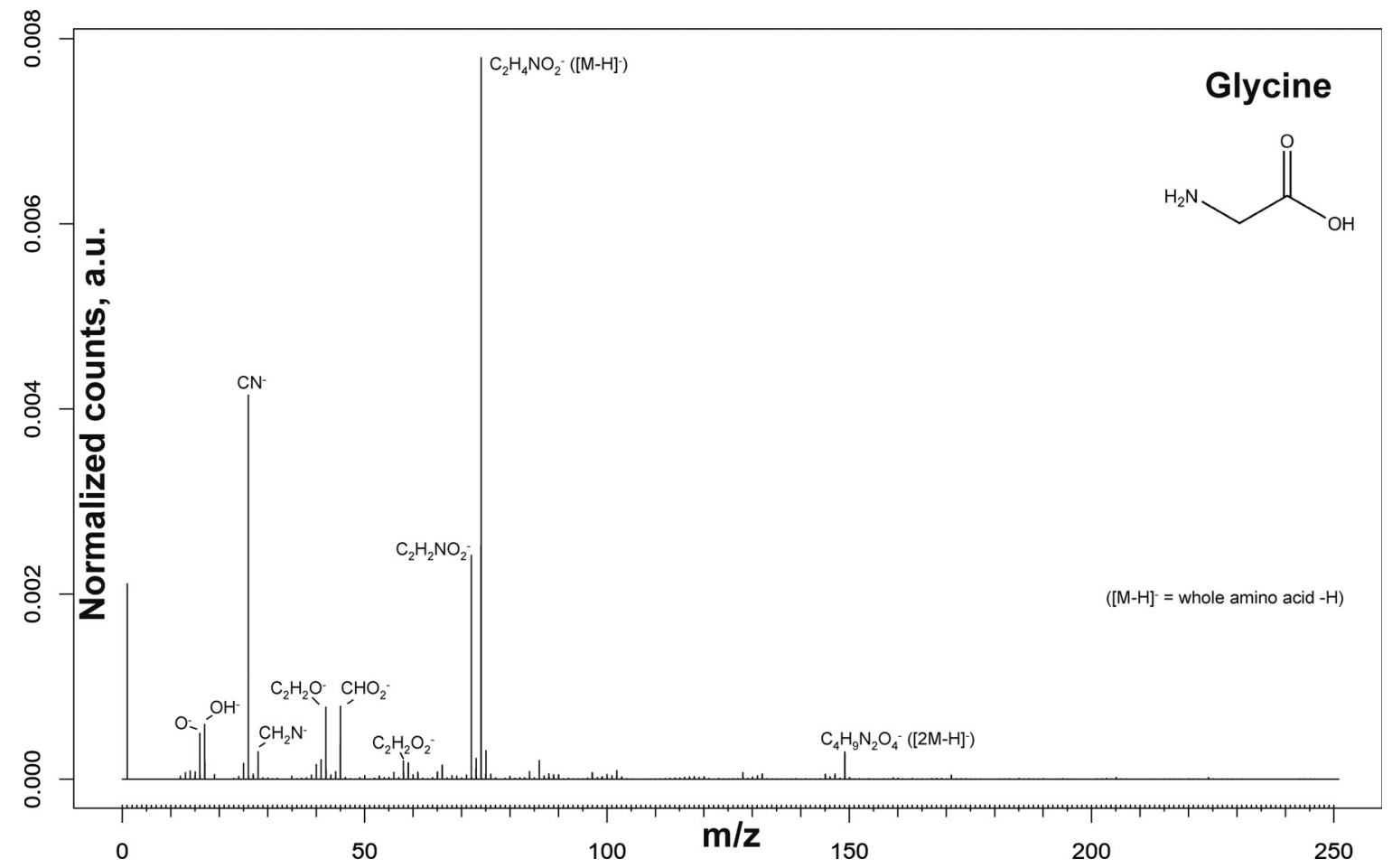

Accession \#

Host Material

- Technique

Secondary Source Polarity

Mass Range Minimum

Mass Range Maximum

Species Used for Mass Calibration

Primary Ion Dose

Primary Species

Primary lon Pulse Width

Pulsed Beam Current

Beam Raster Size
01434-01, 01434-02, 01434-03, 01434-04

Silicon wafer

SIMS

Negative

$0 \mathrm{Da}$

$250 \mathrm{Da}$

$\mathrm{CH}^{-}, \mathrm{OH}^{-}, \mathrm{C}_{2} \mathrm{H}^{-}, \mathrm{CH}_{2} \mathrm{~N}^{-}, \mathrm{C}_{2} \mathrm{H}_{5} \mathrm{NO}_{2}{ }^{-}$

$5.11 \times 10^{11} \mathrm{~cm}^{-2}$

$\mathrm{Bi}_{3}{ }^{+}$

$29.3 \mathrm{~ns}$

$0.0004 \mathrm{nA}$

$200 \times 200 \mu \mathrm{m}^{2}$ 


\section{HISTIDINE (HYDROCHLORIDE)}

\begin{tabular}{lccc}
\hline Spectrum ID \# & Mass (Da) & Species & Peak Assignment \\
\hline $\mathbf{0 1 4 3 5}$ & 50.0036 & $\mathrm{C}_{3} \mathrm{~N}^{-}$ & Histidine \\
$\ldots$ & 65.0145 & $\mathrm{C}_{3} \mathrm{HN}_{2}{ }^{-}$ & $\ldots$ \\
$\ldots$ & 80.0380 & $\mathrm{C}_{4} \mathrm{H}_{4} \mathrm{~N}_{2}^{-}$ & $\ldots$ \\
$\ldots$ & 81.0458 & $\mathrm{C}_{4} \mathrm{H}_{5} \mathrm{~N}_{2}^{-}$ & $\ldots$ \\
$\ldots$ & 93.0458 & $\mathrm{C}_{5} \mathrm{H}_{5} \mathrm{~N}_{2}^{-}$ & $\ldots$ \\
$\ldots$ & 104.0254 & $\mathrm{C}_{5} \mathrm{H}_{2} \mathrm{~N}_{3}^{-}$ & $\ldots$ \\
$\ldots$ & 108.0567 & $\mathrm{C}_{5} \mathrm{H}_{6} \mathrm{~N}_{3}^{-}$ & $\ldots$ \\
$\ldots$ & 137.0357 & $\mathrm{C}_{6} \mathrm{H}_{5} \mathrm{~N}_{2} \mathrm{O}_{2}^{-}$ & $\ldots$ \\
\hline
\end{tabular}

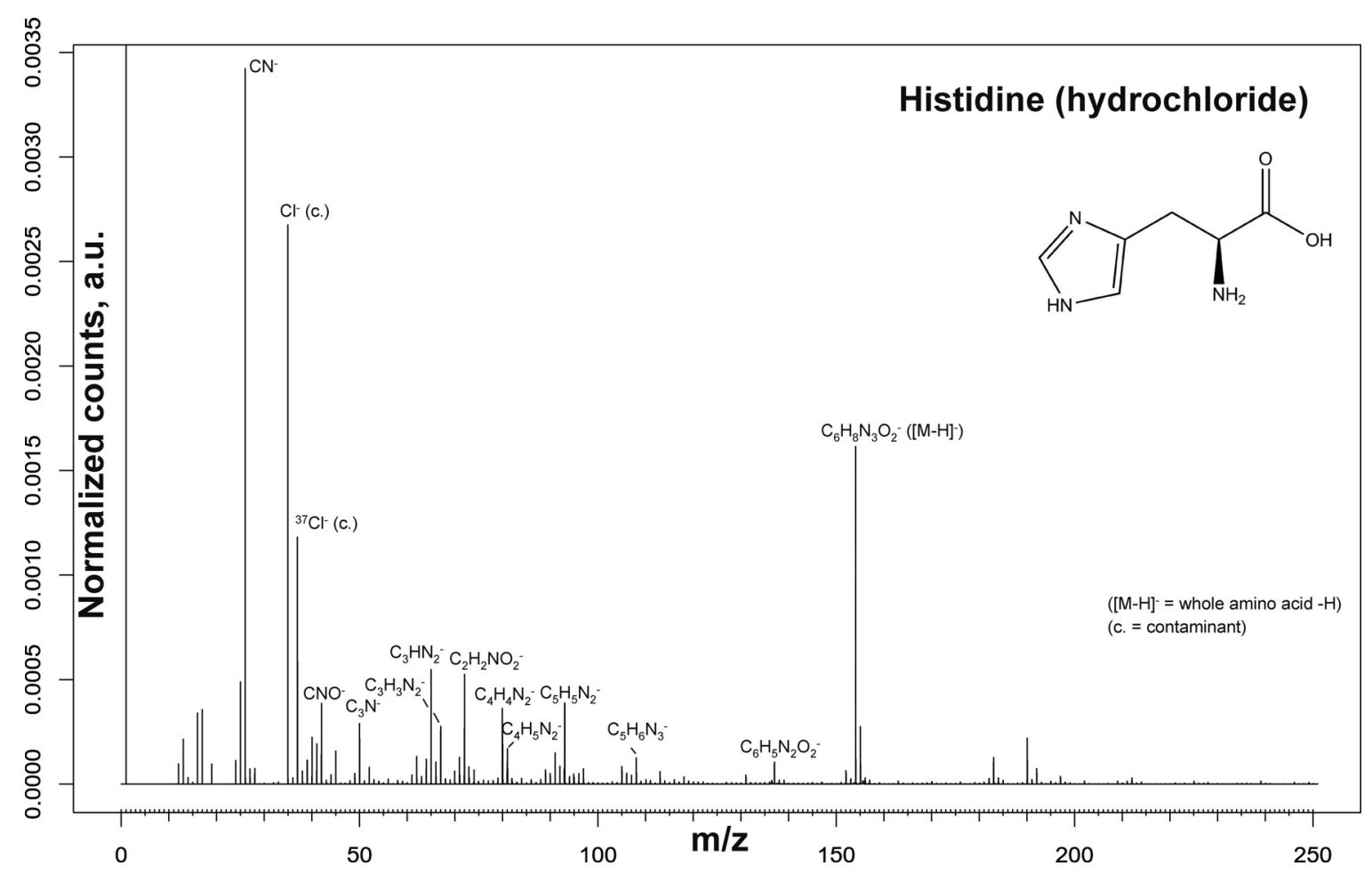

Accession \#

Host Material

- Technique

Secondary Source Polarity

Mass Range Minimum

Mass Range Maximum

Species Used for Mass Calibration

Primary Ion Dose

Primary Species

Primary lon Pulse Width

Pulsed Beam Current

Beam Raster Size
01435-01, 01435-02, 01435-03, 01435-04

Silicon wafer

SIMS

Negative

$0 \mathrm{Da}$

$250 \mathrm{Da}$

$\mathrm{CH}^{-}, \mathrm{OH}^{-}, \mathrm{C}_{2} \mathrm{H}^{-}, \mathrm{CH}_{2} \mathrm{~N}^{-}, \mathrm{C}_{6} \mathrm{H}_{9} \mathrm{~N}_{3} \mathrm{O}_{2}{ }^{-}$

$5.11 \times 10^{11} \mathrm{~cm}^{-2}$

$\mathrm{Bi}_{3}{ }^{+}$

$29.3 \mathrm{~ns}$

$0.0004 \mathrm{nA}$

$200 \times 200 \mu \mathrm{m}^{2}$ 


\begin{tabular}{lccc}
\hline & \multicolumn{3}{c}{ HYDROXYPROLINE } \\
\hline Spectrum ID \# & Mass (Da) & Species & Peak Assignment \\
\hline $\mathbf{0 1 4 3 6}$ & 65.9985 & $\mathrm{C}_{3} \mathrm{NO}^{-}$ & Hydroxyproline \\
$\ldots$ & 66.0349 & $\mathrm{C}_{4} \mathrm{H}_{4} \mathrm{~N}^{-}$ & $\ldots$ \\
$\ldots$ & 71.0139 & $\mathrm{C}_{3} \mathrm{H}_{3} \mathrm{O}_{2}^{-}$ & $\ldots$ \\
$\ldots$ & 72.0091 & $\mathrm{C}_{2} \mathrm{H}_{2} \mathrm{NO}_{2}^{-}$ & $\ldots$ \\
$\ldots$ & 82.0298 & $\mathrm{C}_{4} \mathrm{H}_{4} \mathrm{NO}^{-}$ & $\ldots$ \\
$\ldots$ & 86.0248 & $\mathrm{C}_{3} \mathrm{H}_{4} \mathrm{NO}_{2}{ }^{-}$ & $\ldots$ \\
$\ldots$ & 99.0088 & $\mathrm{C}_{4} \mathrm{H}_{3} \mathrm{O}_{3}^{-}$ & $\ldots$ \\
$\ldots$ & 110.0248 & $\mathrm{C}_{5} \mathrm{H}_{4} \mathrm{NO}_{2}^{-}$ & $\ldots$ \\
$\ldots$ & 128.0353 & $\mathrm{C}_{5} \mathrm{H}_{6} \mathrm{NO}_{3}^{-}$ & $\ldots$ \\
$\ldots$ & 130.0510 & $\mathrm{C}_{5} \mathrm{H}_{8} \mathrm{NO}_{3}{ }^{-}$ & $\ldots$ \\
\hline
\end{tabular}

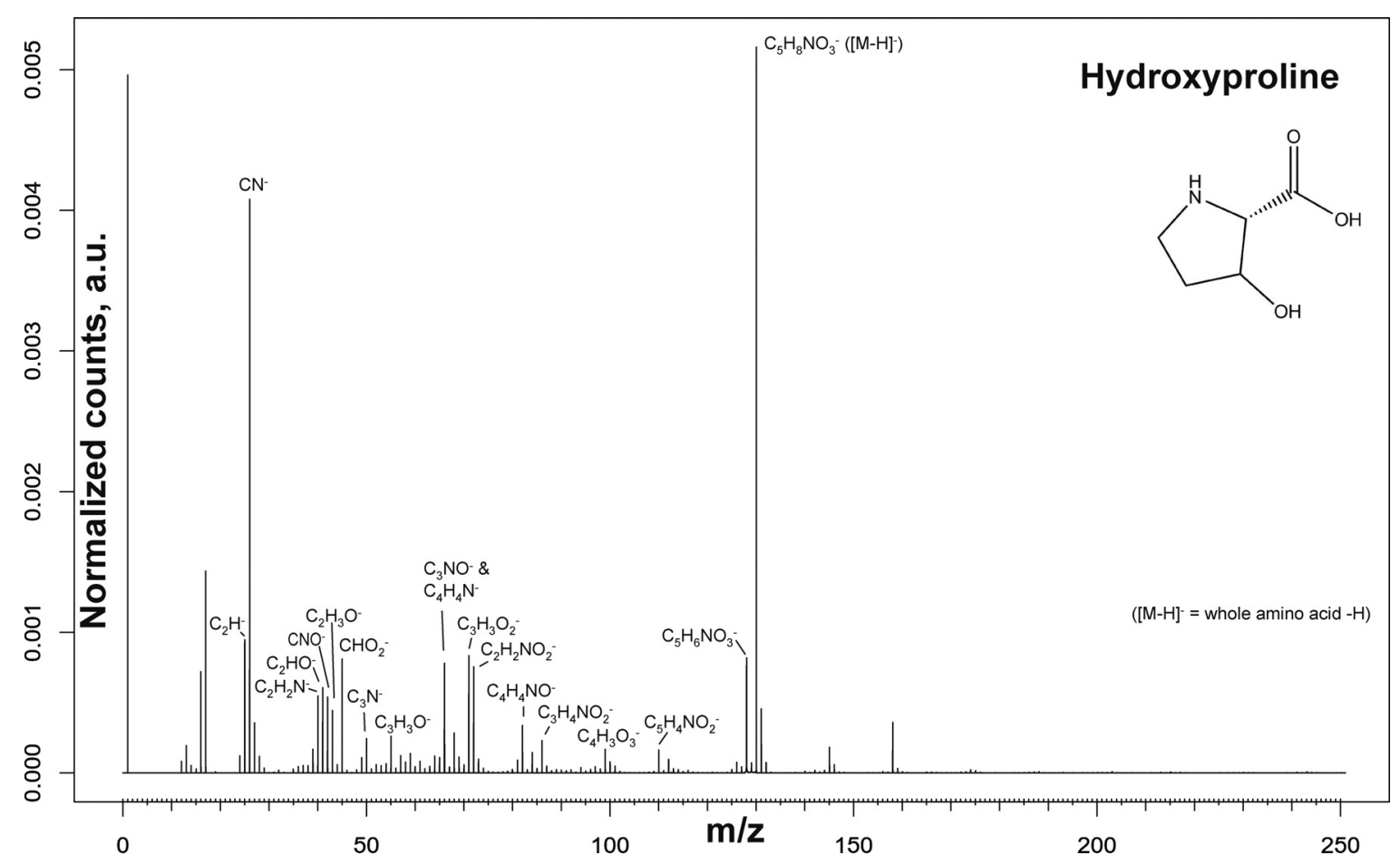

Accession \#

Host Material

- Technique

Secondary Source Polarity

Mass Range Minimum

Mass Range Maximum

Species Used for Mass Calibration

Primary Ion Dose

Primary Species

Primary lon Pulse Width

Pulsed Beam Current

Beam Raster Size
01436-01, 01436-02, 01436-03, 01436-04

Silicon wafer

SIMS

Negative

$0 \mathrm{Da}$

$250 \mathrm{Da}$

$\mathrm{CH}^{-}, \mathrm{O}^{-}, \mathrm{C}_{2} \mathrm{H}^{-}, \mathrm{CH}_{2} \mathrm{~N}^{-}, \mathrm{C}_{5} \mathrm{H}_{9} \mathrm{NO}_{3}^{-}$

$5.11 \times 10^{11} \mathrm{~cm}^{-2}$

$\mathrm{Bi}_{3}{ }^{+}$

$29.3 \mathrm{~ns}$

$0.0004 \mathrm{nA}$

$200 \times 200 \mu \mathrm{m}^{2}$ 


\begin{tabular}{lccc}
\hline & \multicolumn{2}{c}{ ISOLEUCINE } & \\
\hline Spectrum ID \# & Mass (Da) & Species & Peak Assignment \\
\hline $\mathbf{0 1 4 3 7}$ & 72.0091 & $\mathrm{C}_{2} \mathrm{H}_{2} \mathrm{NO}_{2}^{-}$ & Isoleucine \\
$\ldots$ & 85.0169 & $\mathrm{C}_{3} \mathrm{H}_{3} \mathrm{NO}_{2}^{-}$ & $\ldots$ \\
$\ldots$ & 100.0404 & $\mathrm{C}_{4} \mathrm{H}_{6} \mathrm{NO}_{2}^{-}$ & $\ldots$ \\
$\ldots$ & 114.0560 & $\mathrm{C}_{5} \mathrm{H}_{8} \mathrm{NO}_{2}{ }^{-}$ & $\ldots$ \\
$\ldots$ & 128.0717 & $\mathrm{C}_{6} \mathrm{H}_{10} \mathrm{NO}_{2}^{-}$ & $\ldots$ \\
$\ldots$ & 130.0874 & $\mathrm{C}_{6} \mathrm{H}_{12} \mathrm{NO}_{2}^{-}$ & $\ldots$ \\
\hline
\end{tabular}

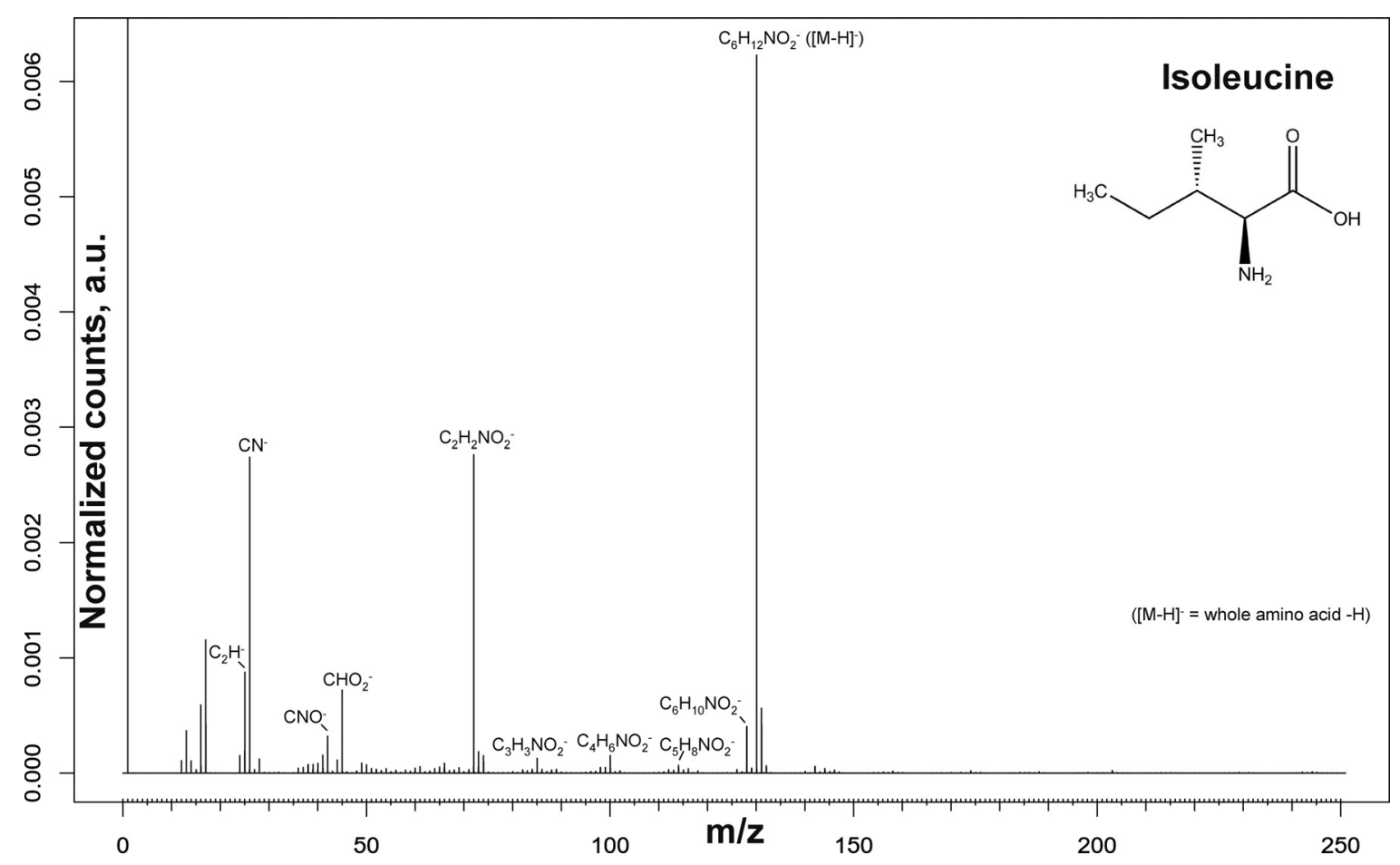

Accession \#

01437-01, 01437-02, 01437-03, 01437-04

Host Material

- Technique

Secondary Source Polarity

Mass Range Minimum

Mass Range Maximum

Species Used for Mass Calibration

Primary lon Dose

Primary Species

Primary lon Pulse Width

Pulsed Beam Current

Beam Raster Size
Silicon wafer

SIMS

Negative

$0 \mathrm{Da}$

$250 \mathrm{Da}$

$\mathrm{CH}^{-}, \mathrm{O}^{-}, \mathrm{C}_{2} \mathrm{H}^{-}, \mathrm{CH}_{2} \mathrm{~N}^{-}, \mathrm{C}_{6} \mathrm{H}_{13} \mathrm{NO}_{2}^{-}$

$5.11 \times 10^{11} \mathrm{~cm}^{-2}$

$\mathrm{Bi}_{3}{ }^{+}$

$29.3 \mathrm{~ns}$

$0.0004 \mathrm{nA}$

$200 \times 200 \mu \mathrm{m}^{2}$ 


\begin{tabular}{lccc}
\hline & \multicolumn{3}{c}{ LEUCINE } \\
\hline Spectrum ID \# & Mass (Da) & Species & Peak Assignment \\
\hline $\mathbf{0 1 4 3 8}$ & 72.0091 & $\mathrm{C}_{2} \mathrm{H}_{2} \mathrm{NO}_{2}^{-}$ & Leucine \\
$\ldots$ & 86.0248 & $\mathrm{C}_{3} \mathrm{H}_{4} \mathrm{NO}_{2}^{-}$ & $\ldots$ \\
$\ldots$ & 114.0560 & $\mathrm{C}_{5} \mathrm{H}_{8} \mathrm{NO}_{2}^{-}$ & $\ldots$ \\
$\ldots$ & 128.0717 & $\mathrm{C}_{6} \mathrm{H}_{10} \mathrm{NO}_{2}^{-}$ & $\ldots$ \\
$\ldots$ & 130.0874 & $\mathrm{C}_{6} \mathrm{H}_{12} \mathrm{NO}_{2}^{-}$ & $\ldots$ \\
\hline
\end{tabular}

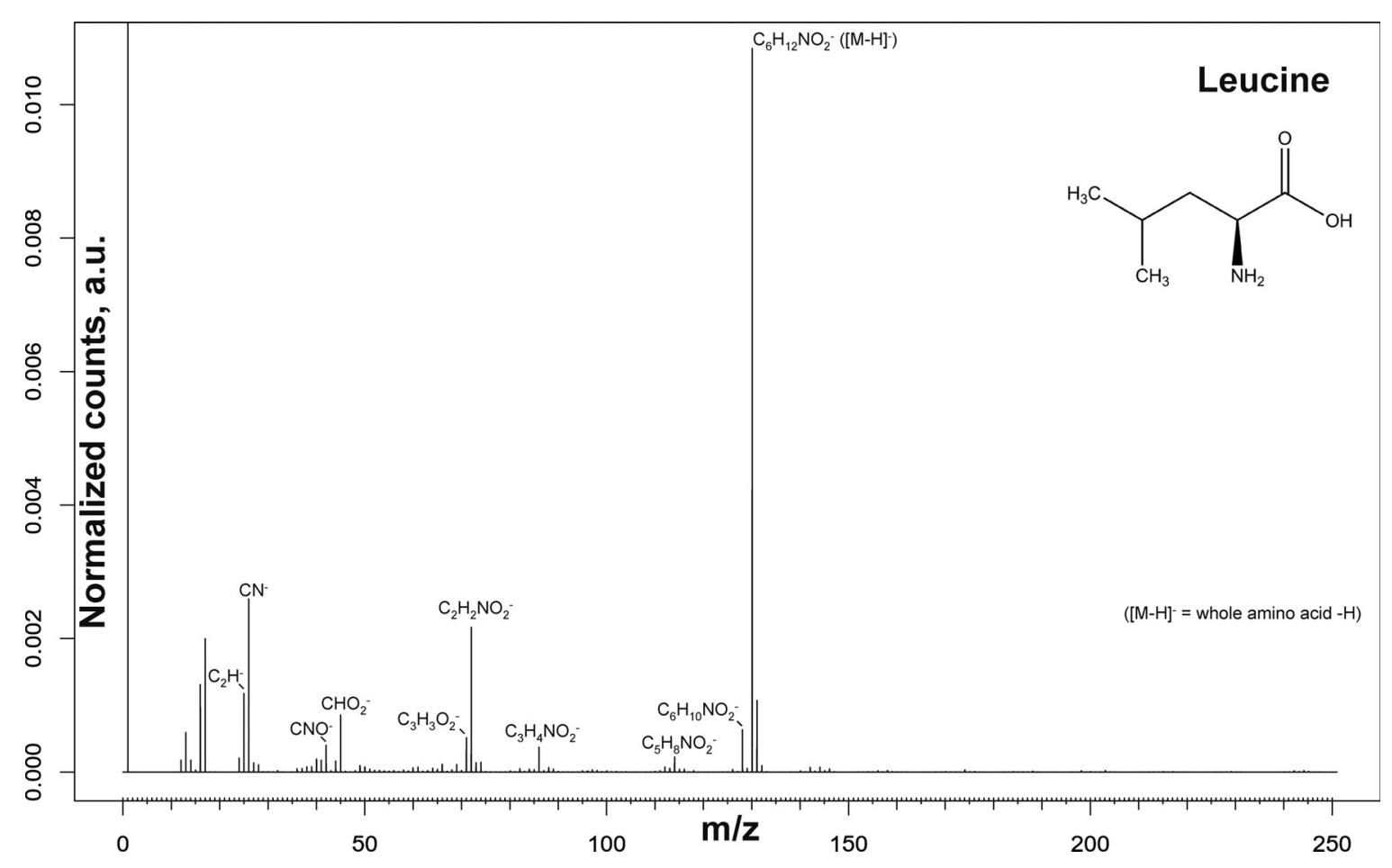

Accession \#

Host Material

- Technique

Secondary Source Polarity

Mass Range Minimum

Mass Range Maximum

Species Used for Mass Calibration

Primary lon Dose

Primary Species

Primary lon Pulse Width

Pulsed Beam Current

Beam Raster Size
01438-01, 01438-02, 01438-03, 01438-04

Silicon wafer

SIMS

Negative

$0 \mathrm{Da}$

$250 \mathrm{Da}$

$\mathrm{CH}^{-}, \mathrm{OH}^{-}, \mathrm{C}_{2} \mathrm{H}^{-}, \mathrm{CH}_{2} \mathrm{~N}^{-}, \mathrm{C}_{6} \mathrm{H}_{10} \mathrm{NO}_{2}{ }^{-}$

$5.11 \times 10^{11} \mathrm{~cm}^{-2}$

$\mathrm{Bi}_{3}{ }^{+}$

$29.3 \mathrm{~ns}$

$0.0004 \mathrm{nA}$

$200 \times 200 \mu \mathrm{m}^{2}$ 


\section{LYSINE (HYDROCHLORIDE)}

\begin{tabular}{lccc}
\hline Spectrum ID \# & Mass (Da) & Species & Peak Assignment \\
\hline $\mathbf{0 1 4 3 9}$ & 71.0139 & $\mathrm{C}_{3} \mathrm{H}_{3} \mathrm{O}_{2}{ }^{-}$ & Lysine \\
$\ldots$ & 72.0091 & $\mathrm{C}_{2} \mathrm{H}_{2} \mathrm{NO}_{2}{ }^{-}$ & $\ldots$ \\
$\ldots$ & 86.0248 & $\mathrm{C}_{3} \mathrm{H}_{4} \mathrm{NO}_{2}^{-}$ & $\ldots$ \\
$\ldots$ & 128.0717 & $\mathrm{C}_{6} \mathrm{H}_{10} \mathrm{NO}_{2}^{-}$ & $\ldots$ \\
$\ldots$ & 130.0874 & $\mathrm{C}_{6} \mathrm{H}_{12} \mathrm{NO}_{2}^{-}$ & $\ldots$ \\
$\ldots$ & 145.0983 & $\mathrm{C}_{6} \mathrm{H}_{13} \mathrm{~N}_{2} \mathrm{O}_{2}^{-}$ & $\ldots$ \\
\hline
\end{tabular}

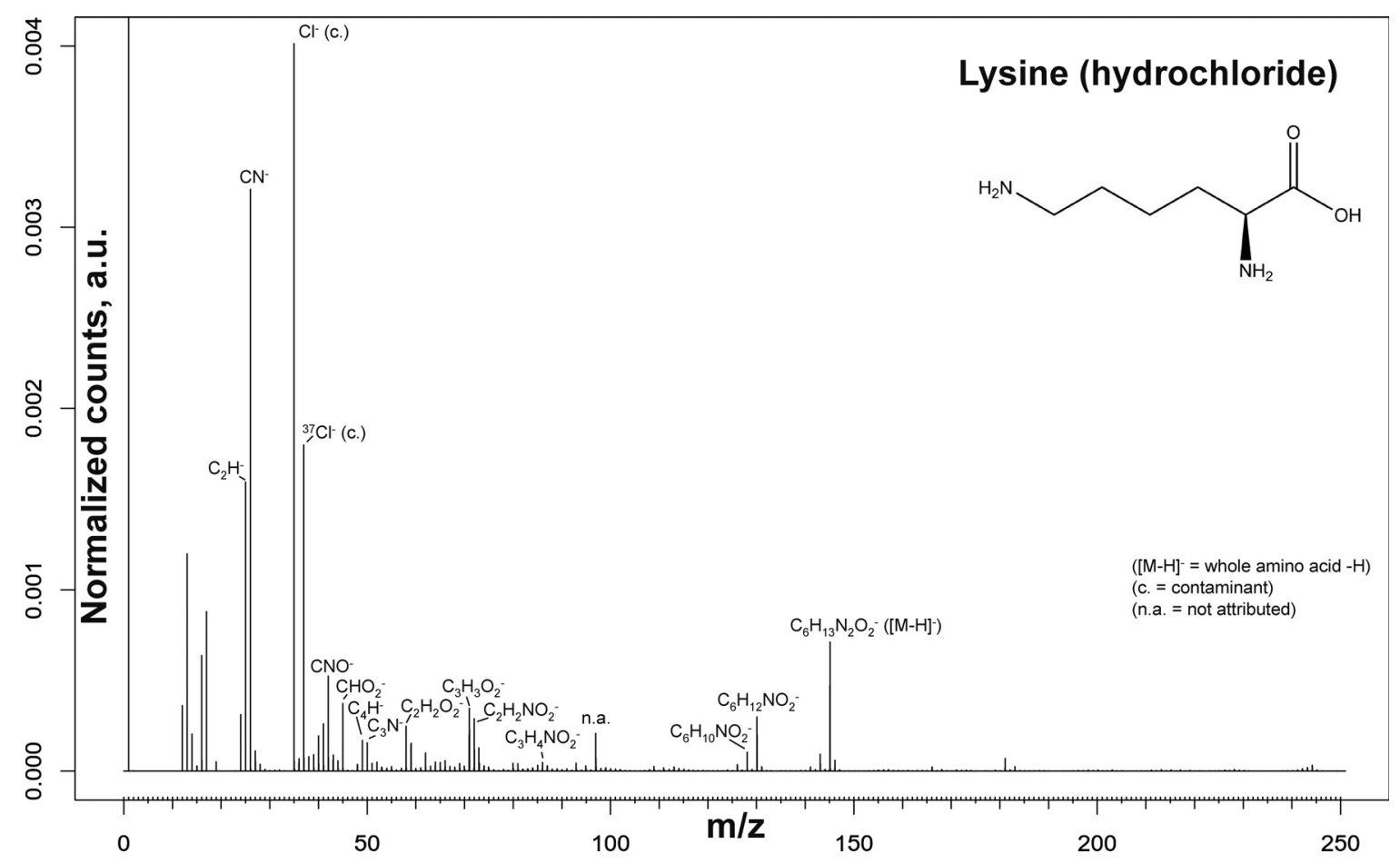

Accession \#

Host Material

- Technique

Secondary Source Polarity

Mass Range Minimum

Mass Range Maximum

Species Used for Mass Calibration

Primary lon Dose

Primary Species

Primary lon Pulse Width

Pulsed Beam Current

Beam Raster Size
01439-01, 01439-02, 01439-03, 01439-04

Silicon wafer

SIMS

Negative

$0 \mathrm{Da}$

$250 \mathrm{Da}$

$\mathrm{CH}^{-}, \mathrm{OH}^{-}, \mathrm{C}_{2} \mathrm{H}^{-}, \mathrm{CH}_{2} \mathrm{~N}^{-}, \mathrm{C}_{6} \mathrm{H}_{14} \mathrm{~N}_{2} \mathrm{O}_{2}^{-}$

$5.11 \times 10^{11} \mathrm{~cm}^{-2}$

$\mathrm{Bi}_{3}{ }^{+}$

$29.3 \mathrm{~ns}$

$0.0004 \mathrm{nA}$

$200 \times 200 \mu \mathrm{m}^{2}$ 


\begin{tabular}{|c|c|c|c|}
\hline \multicolumn{4}{|c|}{ METHIONINE } \\
\hline Spectrum ID \# & Mass (Da) & Species & Peak Assignment \\
\hline 01440 & 31.9726 & $\mathrm{~S}^{-}$ & Methionine \\
\hline$\ldots$ & 32.9804 & $\mathrm{HS}^{-}$ & $\ldots$ \\
\hline$\ldots$ & 46.9961 & $\mathrm{CH}_{3} \mathrm{~S}^{-}$ & $\ldots$ \\
\hline$\ldots$ & 57.9757 & $\mathrm{CNS}^{-}$ & $\ldots$ \\
\hline$\ldots$ & 62.9910 & $\mathrm{CH}_{3} \mathrm{OS}^{-}$ & $\ldots$ \\
\hline$\ldots$ & 72.0091 & $\mathrm{C}_{2} \mathrm{H}_{2} \mathrm{NO}_{2}^{-}$ & $\ldots$ \\
\hline$\ldots$ & 73.0169 & $\mathrm{C}_{2} \mathrm{H}_{3} \mathrm{NO}_{2}^{-}$ & $\ldots$ \\
\hline$\ldots$ & 98.0248 & $\mathrm{C}_{4} \mathrm{H}_{4} \mathrm{NO}_{2}^{-}$ & $\ldots$ \\
\hline$\ldots$ & 100.0404 & $\mathrm{C}_{4} \mathrm{H}_{6} \mathrm{NO}_{2}^{-}$ & $\ldots$ \\
\hline$\ldots$ & 114.9859 & $\mathrm{C}_{4} \mathrm{H}_{3} \mathrm{O}_{2} \mathrm{~S}^{-}$ & $\ldots$ \\
\hline$\ldots$ & 134.0281 & $\mathrm{C}_{4} \mathrm{H}_{8} \mathrm{NO}_{2} \mathrm{~S}^{-}$ & $\ldots$ \\
\hline$\ldots$ & 148.0438 & $\mathrm{C}_{5} \mathrm{H}_{10} \mathrm{NO}_{2} \mathrm{~S}^{-}$ & $\ldots$ \\
\hline$\ldots$ & 164.0387 & $\mathrm{C}_{5} \mathrm{H}_{10} \mathrm{NO}_{3} \mathrm{~S}^{-}$ & $\ldots$ \\
\hline
\end{tabular}

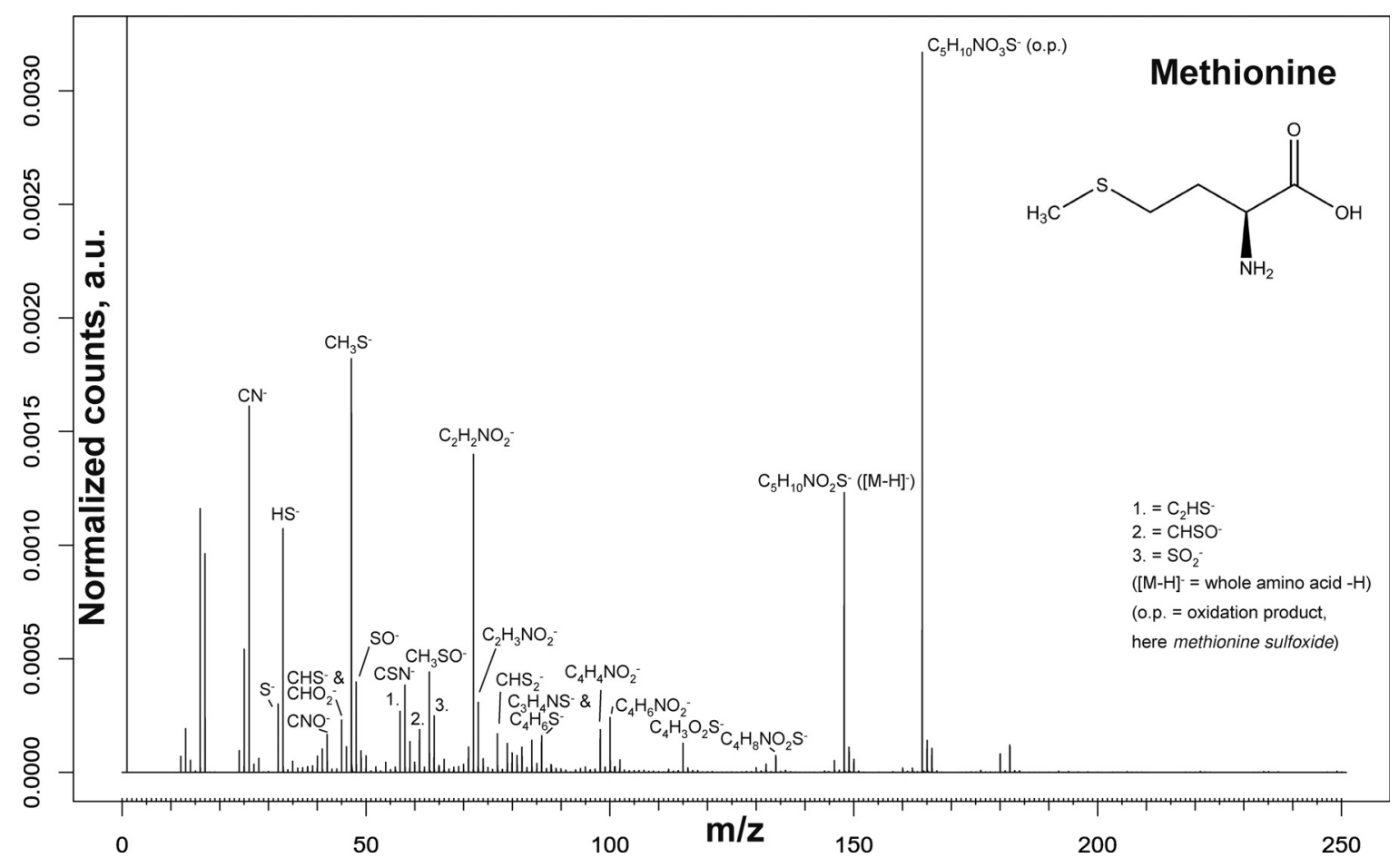

\begin{tabular}{rc}
\hline Accession \# & 01440-01, 01440-02, 01440-03, 01440-04 \\
\hline Host Material & Silicon wafer \\
$\mathbf{\text { Technique }}$ & $\mathrm{SIMS}$ \\
Secondary Source Polarity & $\mathrm{Negative}$ \\
Mass Range Minimum & $0 \mathrm{Da}$ \\
Mass Range Maximum & $250 \mathrm{Da}$ \\
Species Used for Mass Calibration & $\mathrm{CH}^{-}, \mathrm{C}_{2} \mathrm{H}^{-}, \mathrm{CH}_{2} \mathrm{~N}^{-}, \mathrm{C}_{5} \mathrm{H}_{8} \mathrm{NO}_{2} \mathrm{~S}^{-}$ \\
Primary lon Dose & $5.11 \times 10^{11} \mathrm{~cm}^{-2}$ \\
Primary Species & $\mathrm{Bi}_{3}{ }^{+}$ \\
Primary lon Pulse Width & $29.3 \mathrm{~ns}^{-}$ \\
Pulsed Beam Current & $0.0004 \mathrm{nA}^{2}$ \\
Beam Raster Size & $200 \times 200 \mu \mathrm{m}^{2}$ \\
\hline
\end{tabular}




\begin{tabular}{lccc}
\hline & \multicolumn{2}{c}{ PHENYLALANINE } \\
\hline Spectrum ID \# & Mass (Da) & Species & Peak Assignment \\
\hline $\mathbf{0 1 4 4 1}$ & 49.0084 & $\mathrm{C}_{4} \mathrm{H}^{-}$ & Phenylalanine \\
$\ldots$ & 72.0091 & $\mathrm{C}_{2} \mathrm{H}_{2} \mathrm{NO}_{2}^{-}$ & $\ldots$ \\
$\ldots$ & 73.0084 & $\mathrm{C}_{6} \mathrm{H}^{-}$ & $\ldots$ \\
$\ldots$ & 91.0553 & $\mathrm{C}_{7} \mathrm{H}_{7}^{-}$ & $\ldots$ \\
$\ldots$ & 97.0084 & $\mathrm{C}_{8} \mathrm{H}^{-}$ & $\ldots$ \\
$\ldots$ & 103.0553 & $\mathrm{C}_{8} \mathrm{H}_{7}^{-}$ & $\ldots$ \\
$\ldots$ & 118.0662 & $\mathrm{C}_{8} \mathrm{H}_{8} \mathrm{~N}^{-}$ & $\ldots$ \\
$\ldots$ & 147.0452 & $\mathrm{C}_{9} \mathrm{H}_{7} \mathrm{O}_{2}^{-}$ & $\ldots$ \\
$\ldots$ & 164.0717 & $\mathrm{C}_{9} \mathrm{H}_{10} \mathrm{NO}_{2}^{-}$ & $\ldots$ \\
\hline
\end{tabular}

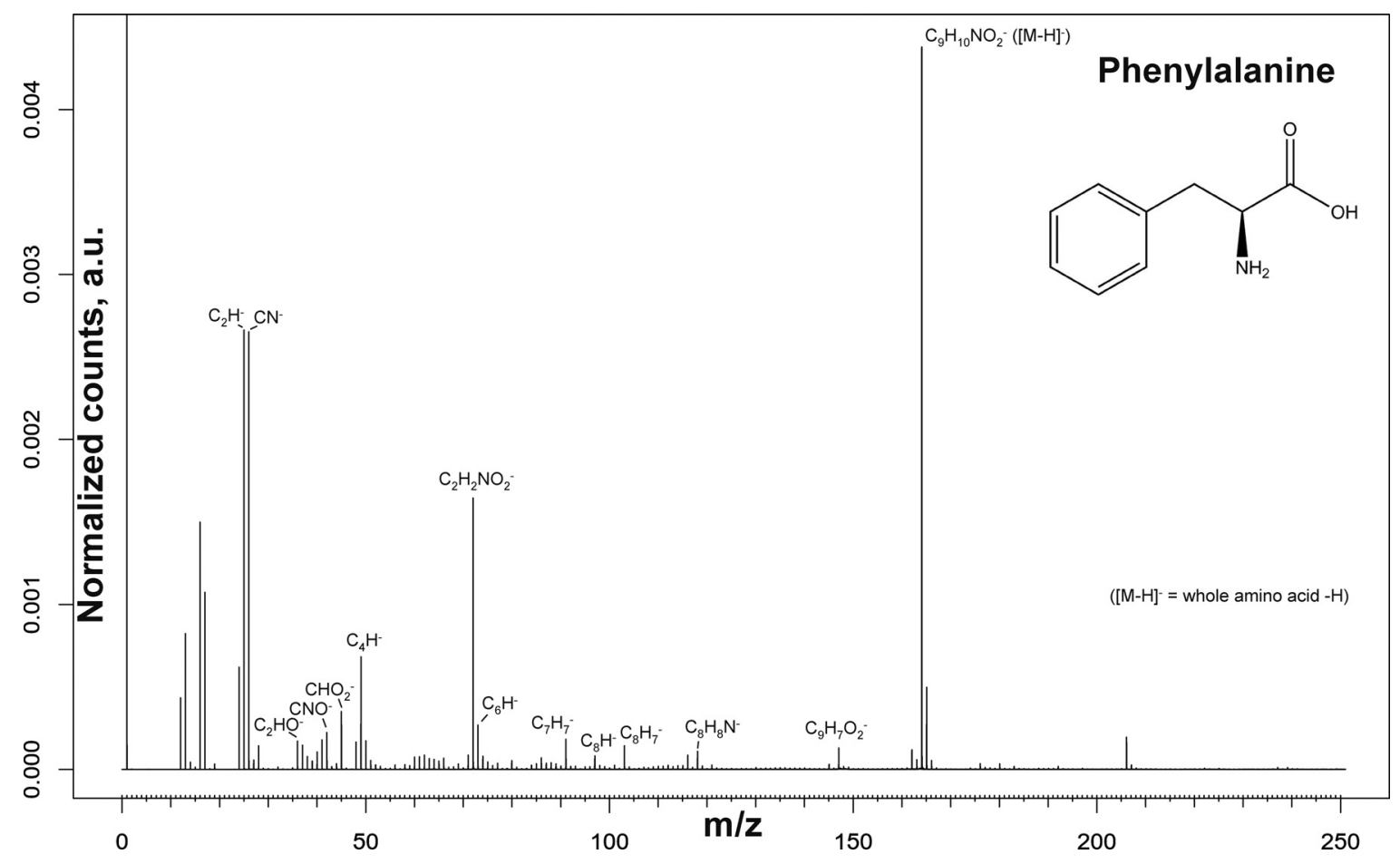

Accession \#

01441-01, 01441-02, 01441-03, 01441-04

Host Material

- Technique

Secondary Source Polarity

Mass Range Minimum

Mass Range Maximum

Species Used for Mass Calibration

Primary lon Dose

Primary Species

Primary lon Pulse Width

Pulsed Beam Current

Beam Raster Size
Silicon wafer

SIMS

Negative

$0 \mathrm{Da}$

$250 \mathrm{Da}$

$\mathrm{CH}^{-}, \mathrm{OH}^{-}, \mathrm{C}_{2} \mathrm{H}^{-}, \mathrm{CH}_{2} \mathrm{~N}^{-}, \mathrm{C}_{9} \mathrm{H}_{11} \mathrm{NO}_{2}{ }^{-}$

$5.11 \times 10^{11} \mathrm{~cm}^{-2}$

$\mathrm{Bi}_{3}{ }^{+}$

29.3 ns

$0.0004 \mathrm{nA}$

$200 \times 200 \mu \mathrm{m}^{2}$ 


\begin{tabular}{lccc}
\hline \multicolumn{3}{c}{ PROLINE } \\
\hline Spectrum ID \# & Mass (Da) & Species & Peak Assignment \\
\hline $\mathbf{0 1 4 4 2}$ & 66.0349 & $\mathrm{C}_{4} \mathrm{H}_{4} \mathrm{~N}^{-}$ & Proline \\
$\ldots$ & 71.0139 & $\mathrm{C}_{3} \mathrm{H}_{3} \mathrm{O}_{2}^{-}$ & $\ldots$ \\
$\ldots$ & 72.0091 & $\mathrm{C}_{2} \mathrm{H}_{2} \mathrm{NO}_{2}^{-}$ & $\ldots$ \\
$\ldots$ & 112.0404 & $\mathrm{C}_{5} \mathrm{H}_{6} \mathrm{NO}_{2}^{-}$ & $\ldots$ \\
$\ldots$ & 114.0560 & $\mathrm{C}_{5} \mathrm{H}_{8} \mathrm{NO}_{2}^{-}$ & $\ldots$ \\
\hline
\end{tabular}

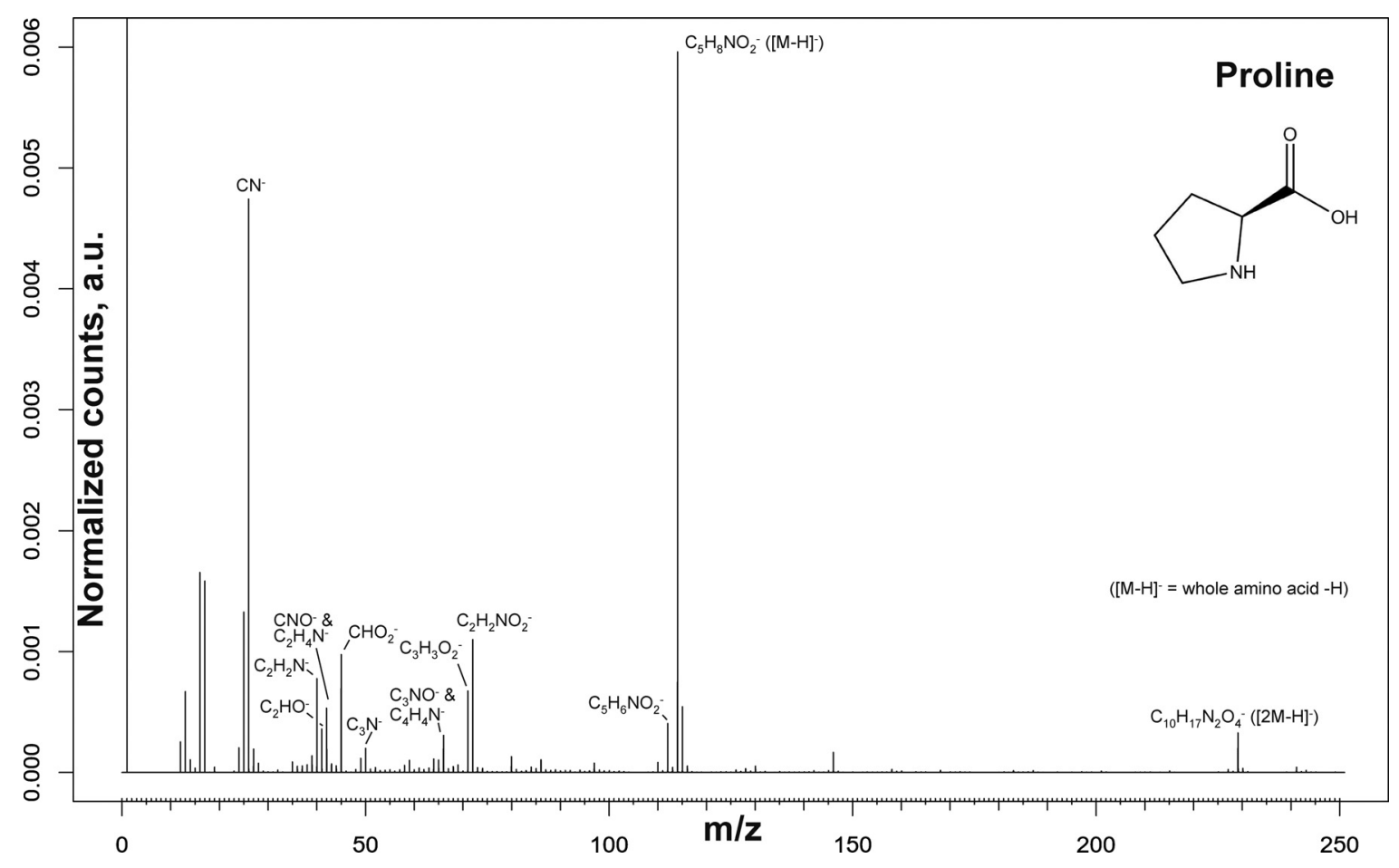

Accession \#

Host Material

- Technique

Secondary Source Polarity

Mass Range Minimum

Mass Range Maximum

Species Used for Mass Calibration

Primary Ion Dose

Primary Species

Primary lon Pulse Width

Pulsed Beam Current

Beam Raster Size
01442-01, 01442-02, 01442-03, 01442-04

Silicon wafer

SIMS

Negative

$0 \mathrm{Da}$

$250 \mathrm{Da}$

$\mathrm{CH}^{-}, \mathrm{C}_{2}^{-}, \mathrm{CH}_{2} \mathrm{~N}^{-}, \mathrm{C}_{5} \mathrm{H}_{6} \mathrm{NO}_{2}^{-}$

$5.11 \times 10^{11} \mathrm{~cm}^{-2}$

$\mathrm{Bi}_{3}{ }^{+}$

$29.3 \mathrm{~ns}$

$0.0004 \mathrm{nA}$

$200 \times 200 \mu \mathrm{m}^{2}$ 


\begin{tabular}{lccc}
\hline \multicolumn{3}{c}{ SERINE } & \\
\hline Spectrum ID \# & Mass (Da) & Species & Peak Assignment \\
\hline $\mathbf{0 1 4 4 3}$ & 59.0139 & $\mathrm{C}_{2} \mathrm{H}_{3} \mathrm{O}_{2}{ }^{-}$ & Serine \\
$\ldots$ & 71.0139 & $\mathrm{C}_{3} \mathrm{H}_{3} \mathrm{O}_{2}^{-}$ & $\ldots$ \\
$\ldots$ & 74.0248 & $\mathrm{C}_{2} \mathrm{H}_{4} \mathrm{NO}_{2}^{-}$ & $\cdots$ \\
$\ldots$ & 87.0088 & $\mathrm{C}_{3} \mathrm{H}_{3} \mathrm{O}_{3}^{-}$ & $\ldots$ \\
$\ldots$ & 104.0353 & $\mathrm{C}_{3} \mathrm{H}_{6} \mathrm{NO}_{3}^{-}$ & $\ldots$ \\
\hline
\end{tabular}

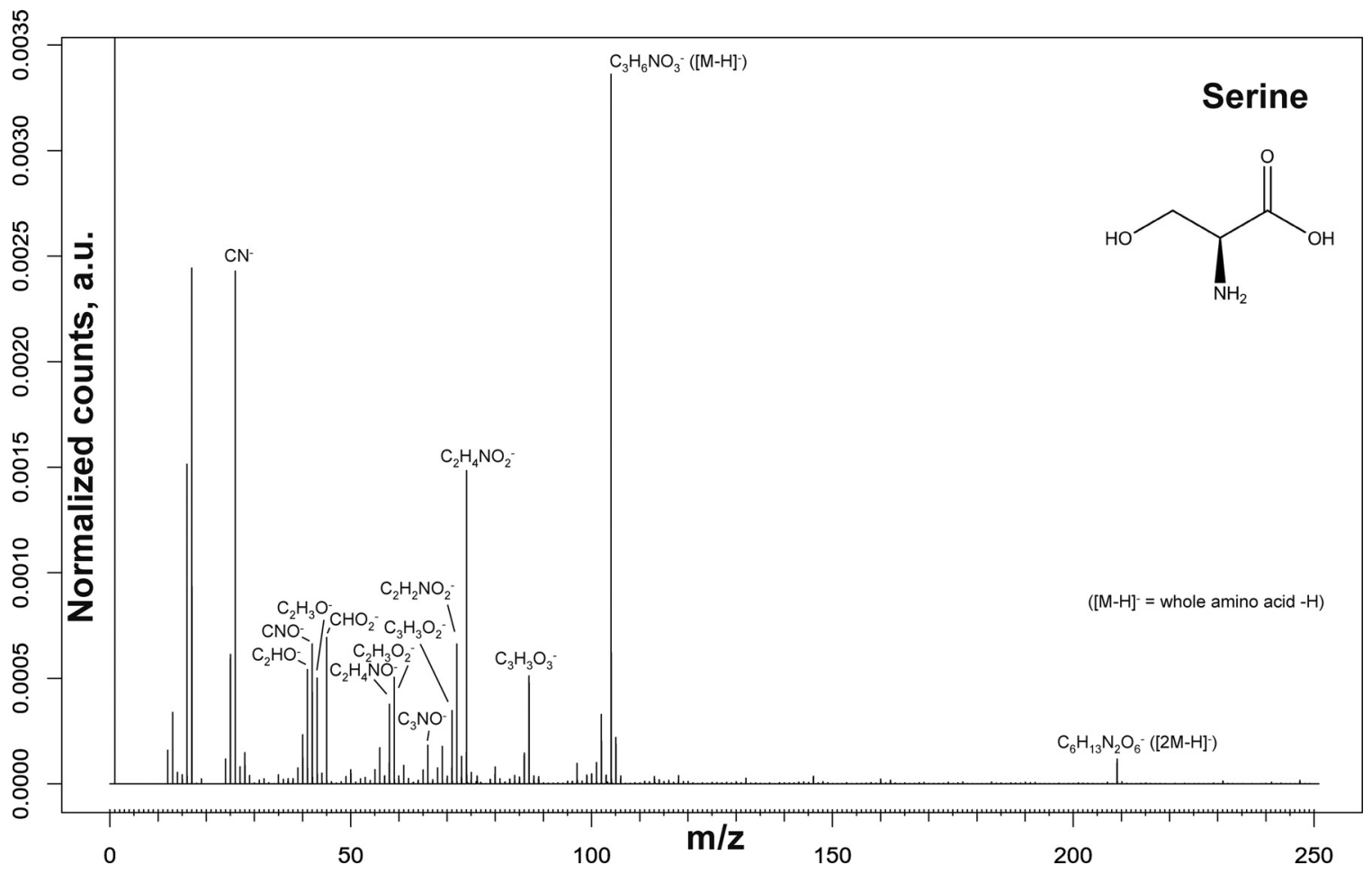

Accession \#

Host Material

- Technique

Secondary Source Polarity

Mass Range Minimum

Mass Range Maximum

Species Used for Mass Calibration

Primary Ion Dose

Primary Species

Primary lon Pulse Width

Pulsed Beam Current

Beam Raster Size
01443-01, 01443-02, 01443-03, 01443-04

Silicon wafer

SIMS

Negative

$0 \mathrm{Da}$

$250 \mathrm{Da}$

$\mathrm{CH}^{-}, \mathrm{O}^{-}, \mathrm{C}_{2} \mathrm{H}^{-}, \mathrm{CH}_{2} \mathrm{~N}^{-}, \mathrm{C}_{3} \mathrm{H}_{7} \mathrm{NO}_{3}{ }^{-}$

$5.11 \times 10^{11} \mathrm{~cm}^{-2}$

$\mathrm{Bi}_{3}{ }^{+}$

$29.3 \mathrm{~ns}$

$0.0004 \mathrm{nA}$

$200 \times 200 \mu \mathrm{m}^{2}$ 


\begin{tabular}{lccc}
\hline \multicolumn{3}{c}{ THREONINE } \\
\hline Spectrum ID \# & Mass (Da) & Species & Peak Assignment \\
\hline $\mathbf{0 1 4 4 4}$ & 72.0091 & $\mathrm{C}_{2} \mathrm{H}_{2} \mathrm{NO}_{2}{ }^{-}$ & Threonine \\
$\ldots$ & 74.0248 & $\mathrm{C}_{2} \mathrm{H}_{4} \mathrm{NO}_{2}{ }^{-}$ & $\ldots$ \\
$\ldots$ & 118.0510 & $\mathrm{C}_{4} \mathrm{H}_{8} \mathrm{NO}_{3}{ }^{-}$ & $\ldots$ \\
\hline
\end{tabular}

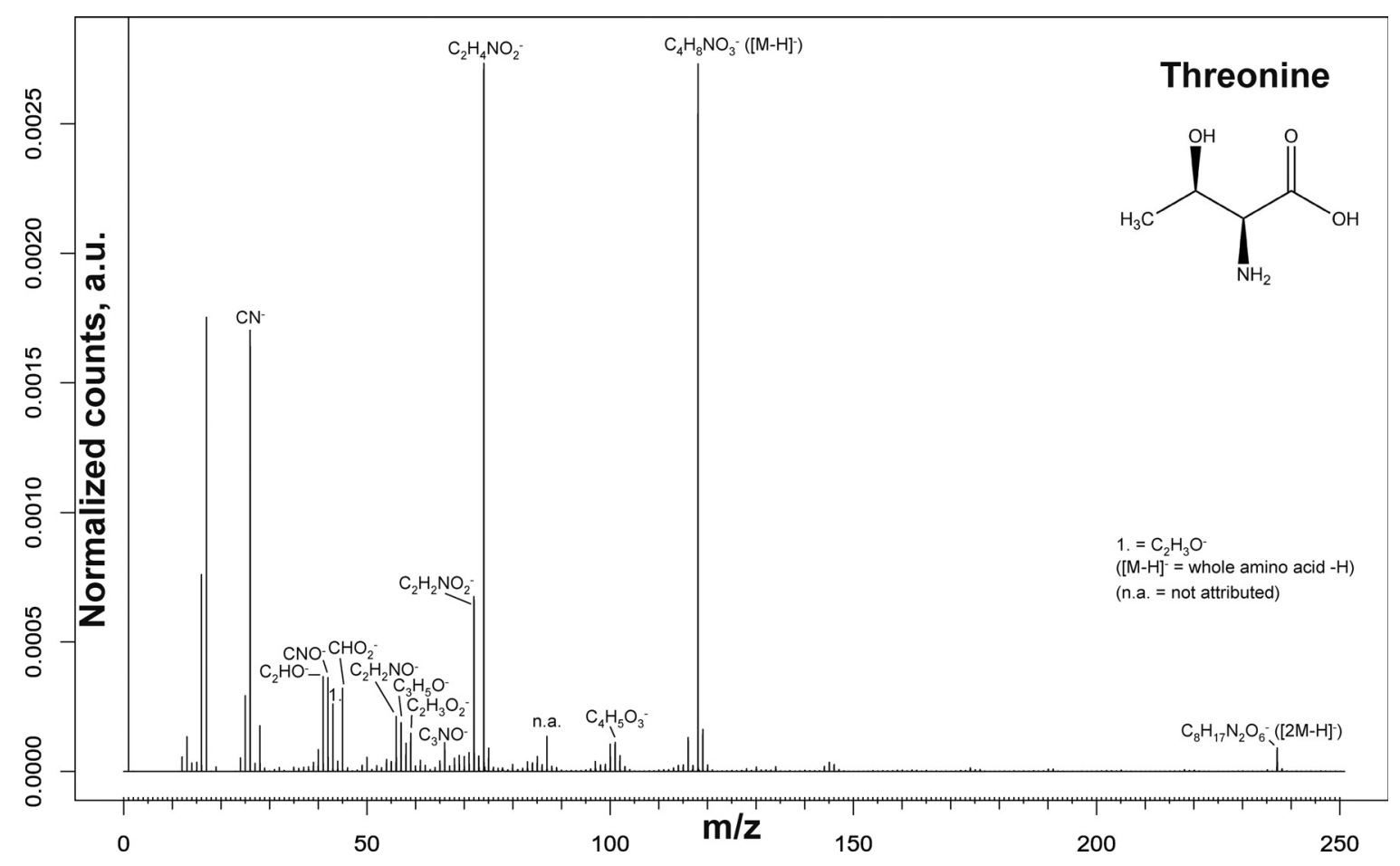

Accession \#

Host Material

- Technique

Secondary Source Polarity

Mass Range Minimum

Mass Range Maximum

Species Used for Mass Calibration

Primary lon Dose

Primary Species

Primary lon Pulse Width

Pulsed Beam Current

Beam Raster Size
01444-01, 01444-02, 01444-03, 01444-04

Silicon wafer

SIMS

Negative

$0 \mathrm{Da}$

$250 \mathrm{Da}$

$\mathrm{CH}^{-}, \mathrm{O}^{-}, \mathrm{C}_{2} \mathrm{H}^{-}, \mathrm{CH}_{2} \mathrm{~N}^{-}, \mathrm{C}_{4} \mathrm{H}_{9} \mathrm{NO}_{3}^{-}$

$5.11 \times 10^{11} \mathrm{~cm}^{-2}$

$\mathrm{Bi}_{3}{ }^{+}$

$29.3 \mathrm{~ns}$

$0.0004 \mathrm{nA}$

$200 \times 200 \mu \mathrm{m}^{2}$ 


\begin{tabular}{lccc}
\hline & \multicolumn{2}{c}{ TRYPTOPHAN } & \\
\hline Spectrum ID \# & Mass (Da) & Species & Peak Assignment \\
\hline $\mathbf{0 1 4 4 5}$ & 49.0084 & $\mathrm{C}_{4} \mathrm{H}^{-}$ & Tryptophan \\
$\ldots$ & 108.0455 & $\mathrm{C}_{6} \mathrm{H}_{6} \mathrm{NO}^{-}$ & $\ldots$ \\
$\ldots$ & 116.0506 & $\mathrm{C}_{8} \mathrm{H}_{6} \mathrm{~N}^{-}$ & $\ldots$ \\
$\ldots$ & 136.0530 & $\mathrm{C}_{8} \mathrm{H}_{8} \mathrm{O}_{2}^{-}$ & $\ldots$ \\
$\ldots$ & 142.0662 & $\mathrm{C}_{10} \mathrm{H}_{8} \mathrm{~N}^{-}$ & $\ldots$ \\
$\ldots$ & 157.0771 & $\mathrm{C}_{10} \mathrm{H}_{9} \mathrm{~N}_{2}^{-}$ & $\ldots$ \\
$\ldots$ & 162.0686 & $\mathrm{C}_{10} \mathrm{H}_{10} \mathrm{O}_{2}^{-}$ & $\ldots$ \\
$\ldots$ & 203.0826 & $\mathrm{C}_{11} \mathrm{H}_{11} \mathrm{~N}_{2} \mathrm{O}_{2}^{-}$ & $\ldots$ \\
\hline
\end{tabular}

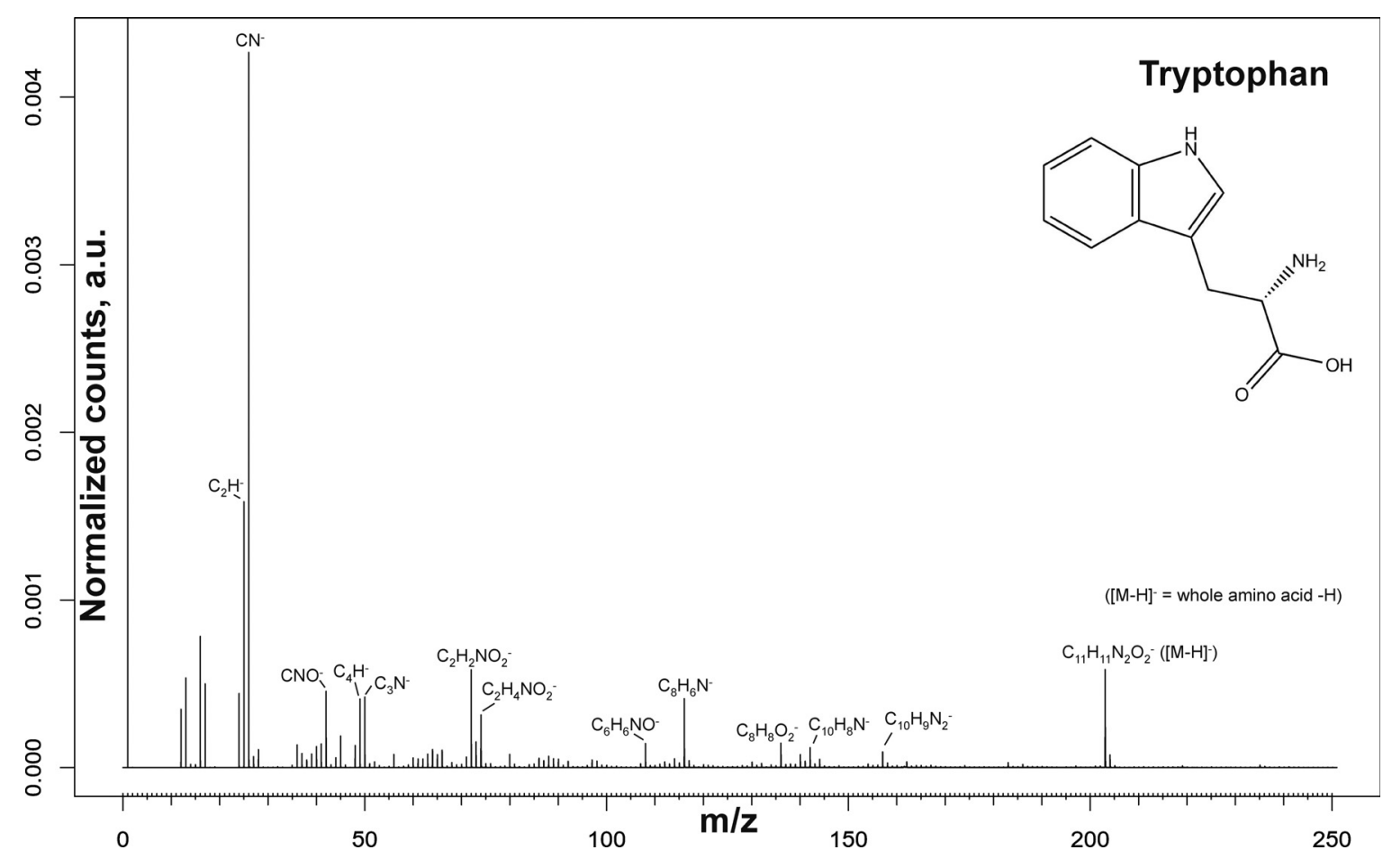

Accession \#

Host Material

- Technique

Secondary Source Polarity

Mass Range Minimum

Mass Range Maximum

Species Used for Mass Calibration

Primary lon Dose

Primary Species

Primary lon Pulse Width

Pulsed Beam Current

Beam Raster Size
01445-01, 01445-02, 01445-03, 01445-04

Silicon wafer

SIMS

Negative

$0 \mathrm{Da}$

$250 \mathrm{Da}$

$\mathrm{CH}^{-}, \mathrm{OH}^{-}, \mathrm{C}_{2} \mathrm{H}^{-}, \mathrm{CH}_{2} \mathrm{~N}^{-}, \mathrm{C}_{11} \mathrm{H}_{12} \mathrm{~N}_{2} \mathrm{O}_{2}^{-}$

$4.73 \times 10^{11} \mathrm{~cm}^{-2}$

$\mathrm{Bi}_{3}{ }^{+}$

$29.3 \mathrm{~ns}$

$0.00037 \mathrm{nA}$

$200 \times 200 \mu \mathrm{m}^{2}$ 


\begin{tabular}{lccc}
\hline & \multicolumn{3}{c}{ TYROSINE } \\
\hline Spectrum ID \# & Mass (Da) & Species & Peak Assignment \\
\hline $\mathbf{0 1 4 4 6}$ & 41.9985 & $\mathrm{CNO}^{-}$ & Tyrosine \\
$\ldots$ & 93.0346 & $\mathrm{C}_{6} \mathrm{H}_{5} \mathrm{O}^{-}$ & $\ldots$ \\
$\ldots$ & 119.0502 & $\mathrm{C}_{8} \mathrm{H}_{7} \mathrm{O}^{-}$ & $\ldots$ \\
$\ldots$ & 134.0611 & $\mathrm{C}_{8} \mathrm{H}_{8} \mathrm{NO}^{-}$ & $\ldots$ \\
$\ldots$ & 163.0401 & $\mathrm{C}_{9} \mathrm{H}_{7} \mathrm{O}_{3}^{-}$ & $\ldots$ \\
$\ldots$ & 180.0666 & $\mathrm{C}_{9} \mathrm{H}_{10} \mathrm{NO}_{3}{ }^{-}$ & $\ldots$ \\
\hline
\end{tabular}

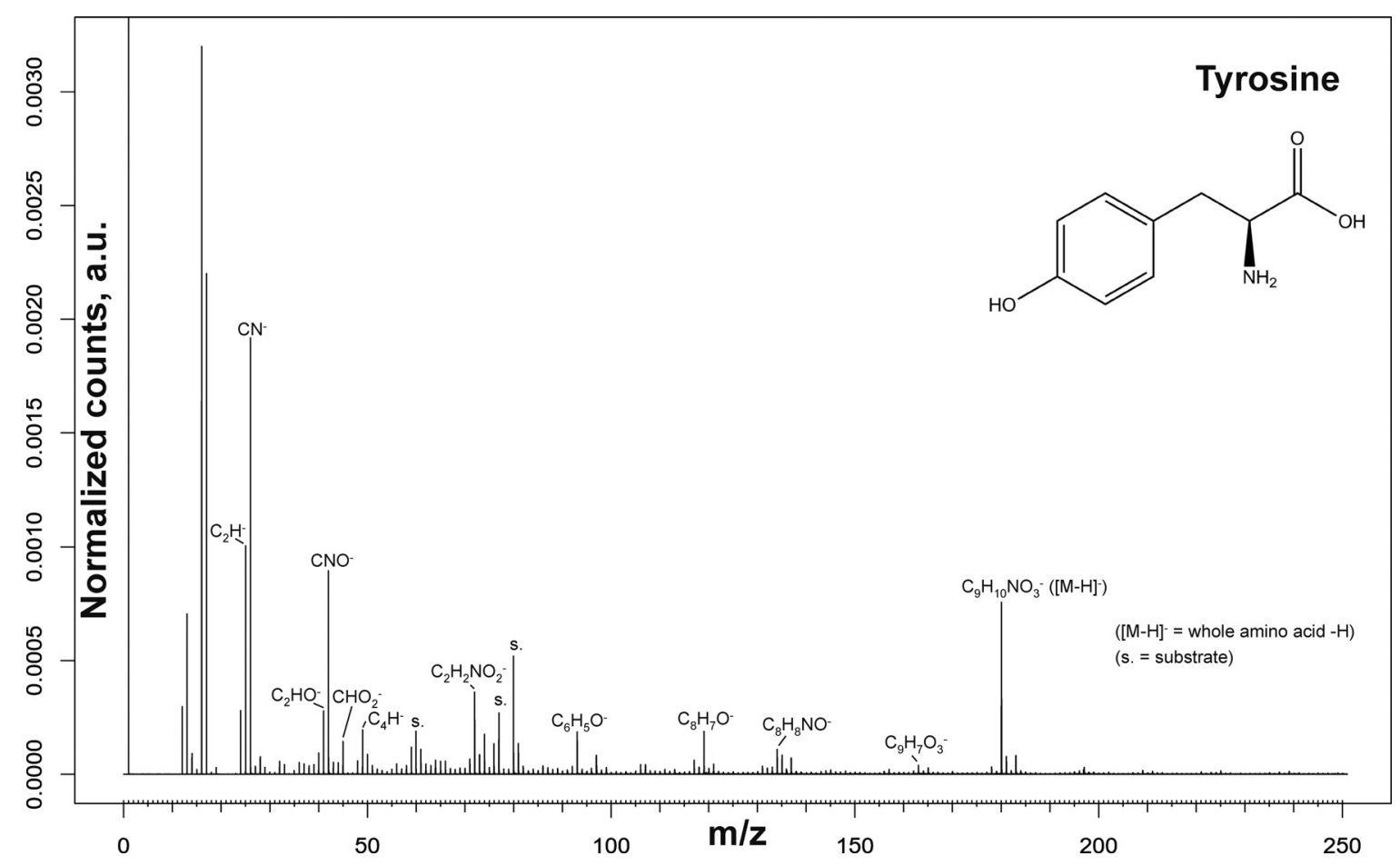

Accession \#

Host Material

Technique

Secondary Source Polarity

Mass Range Minimum

Mass Range Maximum

Species Used for Mass Calibration

Primary Ion Dose

Primary Species

Primary Ion Pulse Width

Pulsed Beam Current

Beam Raster Size

Comment
01446-01, 01446-02, 01446-03, 01446-04

Silicon wafer

SIMS

Negative

$0 \mathrm{Da}$

$250 \mathrm{Da}$

$\mathrm{CH}^{-}, \mathrm{OH}^{-}, \mathrm{C}_{2} \mathrm{H}^{-}, \mathrm{CH}_{2} \mathrm{~N}^{-}, \mathrm{C}_{9} \mathrm{H}_{10} \mathrm{NO}_{3}{ }^{-}$

$4.73 \times 10^{11} \mathrm{~cm}^{-2}$

$\mathrm{Bi}_{3}{ }^{+}$

$29.3 \mathrm{~ns}$

$0.00037 \mathrm{nA}$

$200 \times 200 \mu \mathrm{m}^{2}$

$\mathrm{ROI}$, defined by a minimum threshold of the $\mathrm{C}_{9} \mathrm{H}_{10} \mathrm{NO}_{3}{ }^{-}$signal, was applied to reduce the substrate signal. 


\begin{tabular}{lccc}
\hline & \multicolumn{3}{c}{ VALINE } \\
\hline Spectrum ID \# & Mass (Da) & Species & Peak Assignment \\
\hline $\mathbf{0 1 4 4 7}$ & 72.0091 & $\mathrm{C}_{2} \mathrm{H}_{2} \mathrm{NO}_{2}{ }^{-}$ & Valine \\
$\ldots$ & 85.0295 & $\mathrm{C}_{4} \mathrm{H}_{5} \mathrm{O}_{2}{ }^{-}$ & $\ldots$ \\
$\ldots$ & 100.0404 & $\mathrm{C}_{4} \mathrm{H}_{6} \mathrm{NO}_{2}{ }^{-}$ & $\ldots$ \\
$\ldots$ & 116.0717 & $\mathrm{C}_{5} \mathrm{H}_{10} \mathrm{NO}_{2}{ }^{-}$ & $\ldots$ \\
\hline
\end{tabular}

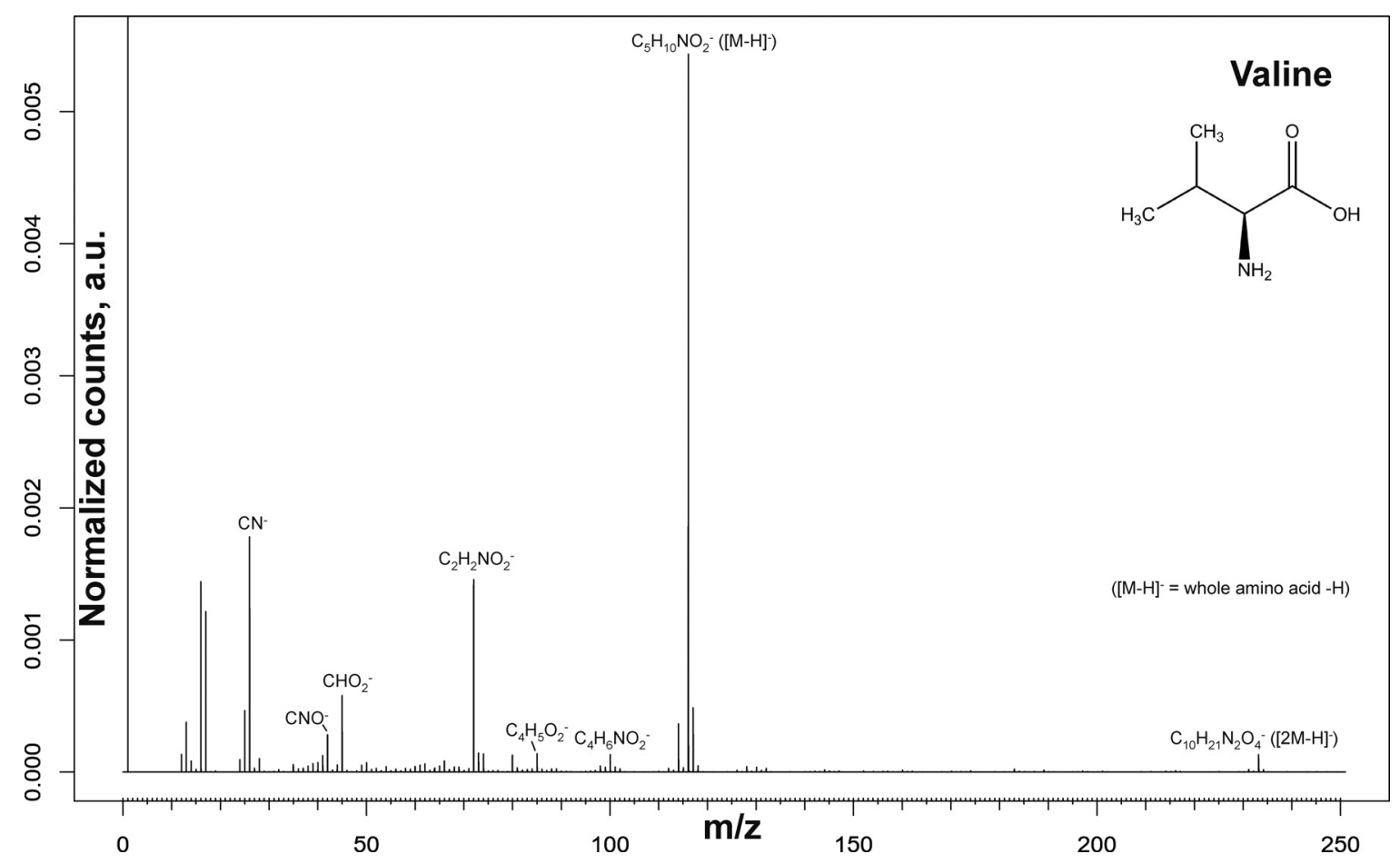

Accession \#

Host Material

- Technique

Secondary Source Polarity

Mass Range Minimum

Mass Range Maximum

Species Used for Mass Calibration

Primary lon Dose

Primary Species

Primary lon Pulse Width

Pulsed Beam Current

Beam Raster Size
01447-01, 01447-02, 01447-03, 01447-04

Silicon wafer

SIMS

Negative

$0 \mathrm{Da}$

$250 \mathrm{Da}$

$\mathrm{CH}^{-}, \mathrm{OH}^{-}, \mathrm{C}_{2} \mathrm{H}^{-}, \mathrm{CH}_{2} \mathrm{~N}^{-}, \mathrm{C}_{5} \mathrm{H}_{11} \mathrm{NO}_{2}{ }^{-}$

$4.73 \times 10^{11} \mathrm{~cm}^{-2}$

$\mathrm{Bi}_{3}{ }^{+}$

$29.3 \mathrm{~ns}$

$0.00037 \mathrm{nA}$

$200 \times 200 \mu \mathrm{m}^{2}$ 

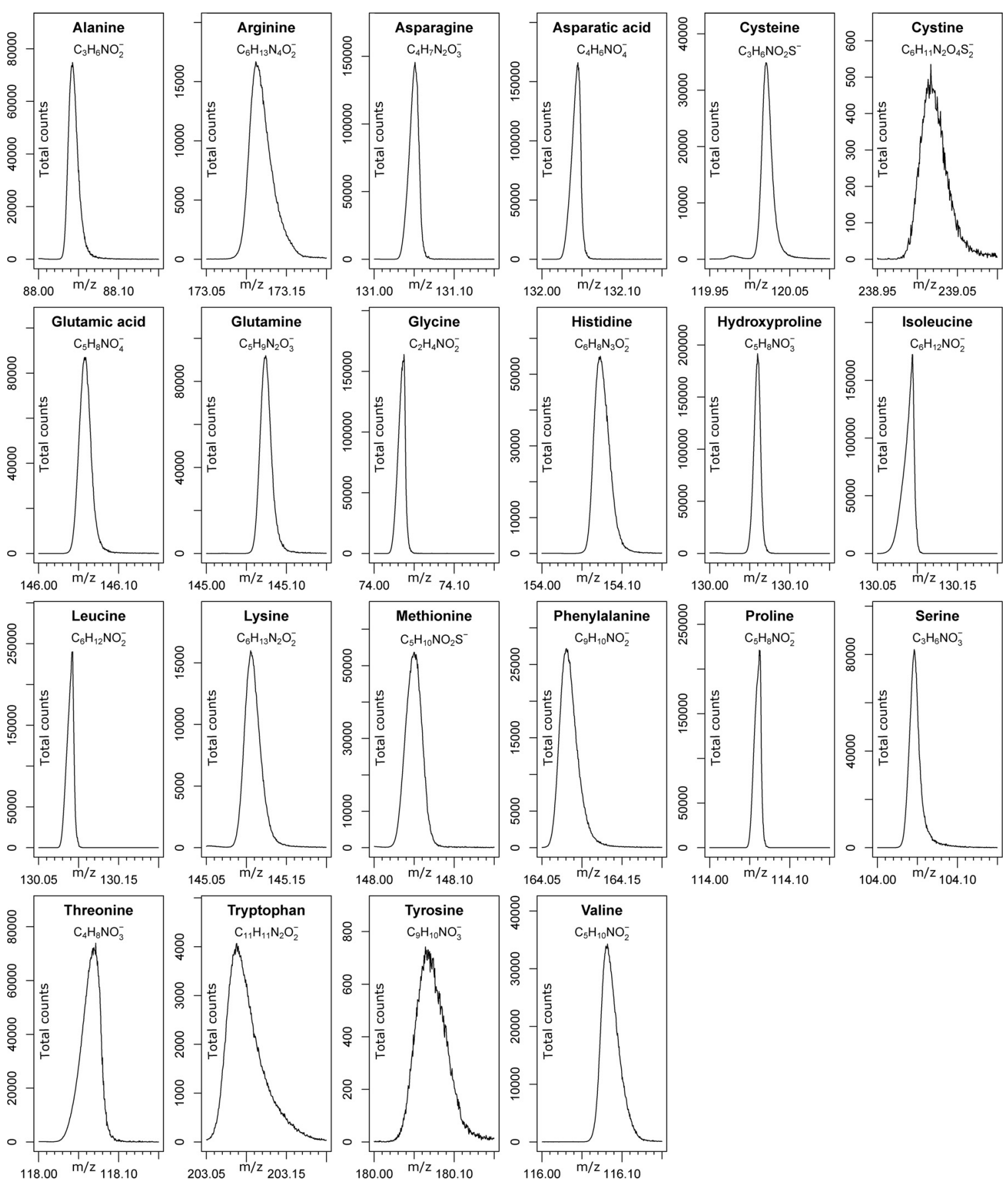

Accession \#

01426-01, 01427-01, 01428-01, 01429-01, 01430-01,

01431-01, 01432-01, 01433-01, 01434-01, 01435-01,

01436-01, 01437-01, 01438-01, 01439-01, 01440-01, 01441-01, 01442-01, 01443-01, 01444-01, 01445-01, 01446-01, 01447-01 


\section{SPECTRAL FEATURES TABLE}

\begin{tabular}{|c|c|c|c|}
\hline $\begin{array}{l}\text { Mass } \\
(\mathrm{Da})\end{array}$ & Peak assignment & Peak present in following amino acids & Peak most prominent in \\
\hline 26.0036 & $\mathrm{CN}^{-}$ & all amino acids & - \\
\hline 31.9726 & $\mathrm{~S}^{-}$ & cysteine, cystine, methionine & cysteine, cystine, methionine \\
\hline 32.9804 & $\mathrm{HS}^{-}$ & cysteine, cystine, methionine & cysteine, cystine, methionine \\
\hline 41.0145 & $\mathrm{CHN}_{2}^{-}$ & arginine, Histidine & arginine \\
\hline 41.9985 & $\mathrm{CNO}^{-}$ & all amino acids & - \\
\hline 42.0111 & $\mathrm{C}_{2} \mathrm{H}_{2} \mathrm{O}^{-}$ & all amino acids & - \\
\hline 42.0349 & $\mathrm{C}_{2} \mathrm{H}_{4} \mathrm{~N}^{-}$ & all amino acids & - \\
\hline 44.9982 & $\mathrm{CHO}_{2}^{-}$ & all amino acids & - \\
\hline 46.9961 & $\mathrm{CH}_{3} \mathrm{~S}^{-}$ & cysteine, cystine, methionine & methionine \\
\hline 49.0084 & $\mathrm{C}_{4} \mathrm{H}^{-}$ & most amino acids & - \\
\hline 50.0036 & $\mathrm{C}_{3} \mathrm{~N}^{-}$ & all amino acids but glycine & - \\
\hline 56.9804 & $\mathrm{C}_{2} \mathrm{HS}^{-}$ & cysteine, cystine, methionine & cysteine, cystine, methionine \\
\hline 57.9757 & $\mathrm{CNS}^{-}$ & cysteine, cystine, methionine & cysteine, cystine, methionine \\
\hline 58.0060 & $\mathrm{C}_{2} \mathrm{H}_{2} \mathrm{O}_{2}^{-}$ & all amino acids & - \\
\hline 58.0298 & $\mathrm{C}_{2} \mathrm{H}_{4} \mathrm{NO}^{-}$ & all amino acids & - \\
\hline 58.0411 & $\mathrm{CH}_{4} \mathrm{~N}_{3}^{-}$ & arginine & arginine \\
\hline 59.0139 & $\mathrm{C}_{2} \mathrm{H}_{3} \mathrm{O}_{2}^{-}$ & all amino acids & - \\
\hline 62.9910 & $\mathrm{CH}_{3} \mathrm{OS}^{-}$ & theoretically none & methionine \\
\hline 63.9447 & $\mathrm{~S}_{2}^{-}$ & cystine & cystine \\
\hline 64.9525 & $\mathrm{HS}_{2}^{-}$ & cystine & cystine \\
\hline 65.0145 & $\mathrm{C}_{3} \mathrm{HN}_{2}^{-}$ & multiple amino acids & histidine \\
\hline 65.9985 & $\mathrm{C}_{3} \mathrm{NO}^{-}$ & all amino acids & - \\
\hline 66.0349 & $\mathrm{C}_{4} \mathrm{H}_{4} \mathrm{~N}^{-}$ & multiple amino acids & hydroxyproline, proline \\
\hline 71.0139 & $\mathrm{C}_{3} \mathrm{H}_{3} \mathrm{O}_{2}^{-}$ & all amino acids but glycine & - \\
\hline 72.0091 & $\mathrm{C}_{2} \mathrm{H}_{2} \mathrm{NO}_{2}^{-}$ & all amino acids & - \\
\hline 73.0084 & $\mathrm{C}_{6} \mathrm{H}^{-}$ & some heavier aminoacids & - \\
\hline 73.0169 & $\mathrm{C}_{2} \mathrm{H}_{3} \mathrm{NO}_{2}^{-}$ & all amino acids & - \\
\hline 74.0070 & $\mathrm{C}_{2} \mathrm{H}_{4} \mathrm{NS}^{-}$ & cysteine, cystine, methionine & cysteine, cystine \\
\hline 74.0248 & $\mathrm{C}_{2} \mathrm{H}_{4} \mathrm{NO}_{2}^{-}$ & all amino acids & - \\
\hline 80.0380 & $\mathrm{C}_{4} \mathrm{H}_{4} \mathrm{~N}_{2}^{-}$ & histidine (and negligibly in some others) & histidine \\
\hline 81.0458 & $\mathrm{C}_{4} \mathrm{H}_{5} \mathrm{~N}_{2}^{-}$ & asparagine, glutamine, histidine & histidine \\
\hline 82.0298 & $\mathrm{C}_{4} \mathrm{H}_{4} \mathrm{NO}^{-}$ & multiple amino acids & - \\
\hline 85.0169 & $\mathrm{C}_{3} \mathrm{H}_{3} \mathrm{NO}_{2}^{-}$ & multiple amino acids & - \\
\hline 85.0295 & $\mathrm{C}_{4} \mathrm{H}_{5} \mathrm{O}_{2}^{-}$ & multiple amino acids & - \\
\hline 86.0248 & $\mathrm{C}_{3} \mathrm{H}_{4} \mathrm{NO}_{2}^{-}$ & multiple amino acids & - \\
\hline 87.0088 & $\mathrm{C}_{3} \mathrm{H}_{3} \mathrm{O}_{3}^{-}$ & hydroxyproline, serine, threonine & serine \\
\hline 88.0404 & $\mathrm{C}_{3} \mathrm{H}_{6} \mathrm{NO}_{2}^{-}$ & multiple amino acids & - \\
\hline 89.0482 & $\mathrm{C}_{3} \mathrm{H}_{7} \mathrm{NO}_{2}^{-}$ & multiple amino acids & - \\
\hline 91.0553 & $\mathrm{C}_{7} \mathrm{H}_{7}^{-}$ & phenylalanine, tryptophan, tyrosine & phenylalanine \\
\hline 93.0346 & $\mathrm{C}_{6} \mathrm{H}_{5} \mathrm{O}^{-}$ & multiple amino acids & - \\
\hline 93.0458 & $\mathrm{C}_{5} \mathrm{H}_{5} \mathrm{~N}_{2}^{-}$ & multiple amino acids & histidine \\
\hline 97.0084 & $\mathrm{C}_{8} \mathrm{H}^{-}$ & phenylalanine, tryptophan, tyrosine & phenylalanine, tryptophan \\
\hline 98.0248 & $\mathrm{C}_{4} \mathrm{H}_{4} \mathrm{NO}_{2}^{-}$ & multiple amino acids & - \\
\hline 99.0088 & $\mathrm{C}_{4} \mathrm{H}_{3} \mathrm{O}_{3}^{-}$ & $\begin{array}{l}\text { asparagine, asparatic acid, } \\
\text { hydroxyproline, threonine }\end{array}$ & - \\
\hline
\end{tabular}




\begin{tabular}{|c|c|c|c|}
\hline $\begin{array}{l}\text { Mass } \\
\text { (Da) }\end{array}$ & Peak assignment & Peak present in following amino acids & Peak most prominent in \\
\hline 99.0564 & $\mathrm{C}_{4} \mathrm{H}_{7} \mathrm{~N}_{2} \mathrm{O}^{-}$ & multiple amino acids & - \\
\hline 100.0404 & $\mathrm{C}_{4} \mathrm{H}_{6} \mathrm{NO}_{2}^{-}$ & multiple amino acids & - \\
\hline 102.0560 & $\mathrm{C}_{4} \mathrm{H}_{8} \mathrm{NO}_{2}^{-}$ & multiple amino acids & - \\
\hline 102.9859 & $\mathrm{C}_{3} \mathrm{H}_{3} \mathrm{O}_{2} \mathrm{~S}^{-}$ & cysteine, cystine & cysteine \\
\hline 103.0553 & $\mathrm{C}_{8} \mathrm{H}_{7}^{-}$ & phenylalanine, tryptophan, tyrosine & phenylalanine \\
\hline 104.0176 & $\mathrm{C}_{3} \mathrm{H}_{6} \mathrm{NOS}^{-}$ & cysteine, cystine & cystine \\
\hline 104.0254 & $\mathrm{C}_{5} \mathrm{H}_{2} \mathrm{~N}_{3}^{-}$ & histidine (and negligeably in some others) & histidine \\
\hline 104.0353 & $\mathrm{C}_{3} \mathrm{H}_{6} \mathrm{NO}_{3}^{-}$ & hydroxyproline, serine, threonine & serine \\
\hline 108.0455 & $\mathrm{C}_{6} \mathrm{H}_{6} \mathrm{NO}^{-}$ & multiple amino acids & - \\
\hline 108.0567 & $\mathrm{C}_{5} \mathrm{H}_{6} \mathrm{~N}_{3}^{-}$ & histidine & histidine \\
\hline 110.0248 & $\mathrm{C}_{5} \mathrm{H}_{4} \mathrm{NO}_{2}^{-}$ & multiple amino acids & - \\
\hline 112.0404 & $\mathrm{C}_{5} \mathrm{H}_{6} \mathrm{NO}_{2}^{-}$ & multiple amino acids & - \\
\hline 113.0357 & $\mathrm{C}_{4} \mathrm{H}_{5} \mathrm{~N}_{2} \mathrm{O}_{2}^{-}$ & glutamic acid, glutamine, asparagine, histidine & asparagine, histidine \\
\hline 114.0197 & $\mathrm{C}_{4} \mathrm{H}_{4} \mathrm{NO}_{3}^{-}$ & asparagine, asparatic acid, hydroxyproline, threonine & asparagine \\
\hline 114.0560 & $\mathrm{C}_{5} \mathrm{H}_{8} \mathrm{NO}_{2}^{-}$ & multiple amino acids & isoleucine, leucine, proline \\
\hline 114.9859 & $\mathrm{C}_{4} \mathrm{H}_{3} \mathrm{O}_{2} \mathrm{~S}^{-}$ & methionine & methionine \\
\hline 115.0037 & $\mathrm{C}_{4} \mathrm{H}_{3} \mathrm{O}_{4}^{-}$ & asparatic acid & asparatic acid \\
\hline 116.0506 & $\mathrm{C}_{8} \mathrm{H}_{6} \mathrm{~N}^{-}$ & phenylalanine, tryptophan & tryptophan \\
\hline 116.0717 & $\mathrm{C}_{5} \mathrm{H}_{10} \mathrm{NO}_{2}^{-}$ & valine & valine \\
\hline 117.0193 & $\mathrm{C}_{4} \mathrm{H}_{5} \mathrm{O}_{4}^{-}$ & asparatic acid & asparatic acid \\
\hline 118.0510 & $\mathrm{C}_{4} \mathrm{H}_{8} \mathrm{NO}_{3}^{-}$ & asparagine, asparatic acid, hydroxyproline, threonine & threonine \\
\hline 118.0662 & $\mathrm{C}_{8} \mathrm{H}_{8} \mathrm{~N}^{-}$ & phenylalanine & phenylalanine \\
\hline 119.0502 & $\mathrm{C}_{8} \mathrm{H}_{7} \mathrm{O}^{-}$ & tyrosine & tyrosine \\
\hline 120.0125 & $\mathrm{C}_{3} \mathrm{H}_{6} \mathrm{NO}_{2} \mathrm{~S}^{-}$ & cysteine, cystine & cysteine, cystine \\
\hline 128.0353 & $\mathrm{C}_{5} \mathrm{H}_{6} \mathrm{NO}_{3}^{-}$ & glutamine, glutamic acid, hydroxyprolin & glutamine, glutamic acid, hydroxyprolin \\
\hline 128.0717 & $\mathrm{C}_{6} \mathrm{H}_{10} \mathrm{NO}_{2}^{-}$ & isoleucine, leucine, lysine & isoleucine, leucine, lysine \\
\hline 130.0510 & $\mathrm{C}_{5} \mathrm{H}_{8} \mathrm{NO}_{3}^{-}$ & glutamic acid, glutamine, hydroxyproline, proline & hydroxyproline \\
\hline 130.0874 & $\mathrm{C}_{6} \mathrm{H}_{12} \mathrm{NO}_{2}^{-}$ & isoleucine, leucine, lysine & isoleucine, leucine, lysine \\
\hline 131.0350 & $\mathrm{C}_{5} \mathrm{H}_{7} \mathrm{O}_{4}^{-}$ & glutamic acid & glutamic acid \\
\hline 131.0462 & $\mathrm{C}_{4} \mathrm{H}_{7} \mathrm{~N}_{2} \mathrm{O}_{3}^{-}$ & asparagine & asparagine \\
\hline 131.0826 & $\mathrm{C}_{5} \mathrm{H}_{11} \mathrm{~N}_{2} \mathrm{O}_{2}^{-}$ & arginine & arginine \\
\hline 132.0302 & $\mathrm{C}_{4} \mathrm{H}_{6} \mathrm{NO}_{4}^{-}$ & asparatic acid & asparatic acid \\
\hline 134.0281 & $\mathrm{C}_{4} \mathrm{H}_{8} \mathrm{NO}_{2} \mathrm{~S}^{-}$ & methionine & methionine \\
\hline 134.0611 & $\mathrm{C}_{8} \mathrm{H}_{8} \mathrm{NO}^{-}$ & tyrosine & tyrosine \\
\hline 136.0530 & $\mathrm{C}_{8} \mathrm{H}_{8} \mathrm{O}_{2}^{-}$ & tryptophan & tryptophan \\
\hline 137.0357 & $\mathrm{C}_{6} \mathrm{H}_{5} \mathrm{~N}_{2} \mathrm{O}_{2}^{-}$ & histidine & histidine \\
\hline 142.0662 & $\mathrm{C}_{10} \mathrm{H}_{8} \mathrm{~N}^{-}$ & tryptophan & tryptophan \\
\hline 145.0619 & $\mathrm{C}_{5} \mathrm{H}_{9} \mathrm{~N}_{2} \mathrm{O}_{3}^{-}$ & glutamine & glutamine \\
\hline 145.0983 & $\mathrm{C}_{6} \mathrm{H}_{13} \mathrm{~N}_{2} \mathrm{O}_{2}^{-}$ & lysine & lysine \\
\hline 146.0459 & $\mathrm{C}_{5} \mathrm{H}_{8} \mathrm{NO}_{4}^{-}$ & glutamic acid & glutamic acid \\
\hline 147.0452 & $\mathrm{C}_{9} \mathrm{H}_{7} \mathrm{O}_{2}^{-}$ & phenylalanine & phenylalanine \\
\hline 148.0438 & $\mathrm{C}_{5} \mathrm{H}_{10} \mathrm{NO}_{2} \mathrm{~S}^{-}$ & methionine & methionine \\
\hline 151.9845 & $\mathrm{C}_{3} \mathrm{H}_{6} \mathrm{NO}_{2} \mathrm{~S}_{2}^{-}$ & cystine & cystine \\
\hline 154.0622 & $\mathrm{C}_{6} \mathrm{H}_{8} \mathrm{~N}_{3} \mathrm{O}_{2}^{-}$ & histidine & histidine \\
\hline 156.0779 & $\mathrm{C}_{6} \mathrm{H}_{10} \mathrm{~N}_{3} \mathrm{O}_{2}^{-}$ & arginine & arginine \\
\hline 157.0771 & $\mathrm{C}_{10} \mathrm{H}_{9} \mathrm{~N}_{2}^{-}$ & tryptophan & tryptophan \\
\hline
\end{tabular}




\begin{tabular}{lccc}
\hline $\begin{array}{l}\text { Mass } \\
\text { (Da) }\end{array}$ & Peak assignment & Peak present in following amino acids & Peak most prominent in \\
\hline 158.0935 & $\mathrm{C}_{6} \mathrm{H}_{12} \mathrm{~N}_{3} \mathrm{O}_{2}^{-}$ & arginine & arginine \\
162.0686 & $\mathrm{C}_{10} \mathrm{H}_{10} \mathrm{O}_{2}^{-}$ & tryptophan & tryptophan \\
163.0401 & $\mathrm{C}_{9} \mathrm{H}_{7} \mathrm{O}_{3}^{-}$ & tyrosine & tyrosine \\
164.0387 & $\mathrm{C}_{5} \mathrm{H}_{10} \mathrm{NO}_{3} \mathrm{~S}^{-}$ & methionine & methionine \\
164.0717 & $\mathrm{C}_{9} \mathrm{H}_{10} \mathrm{NO}_{2}^{-}$ & phenylalanine & phenylalanine \\
173.1044 & $\mathrm{C}_{6} \mathrm{H}_{13} \mathrm{~N}_{4} \mathrm{O}_{2}^{-}$ & arginine & arginine \\
180.0666 & $\mathrm{C}_{9} \mathrm{H}_{10} \mathrm{NO}_{3}^{-}$ & tyrosine & tyrosine \\
203.0826 & $\mathrm{C}_{11} \mathrm{H}_{11} \mathrm{~N}_{2} \mathrm{O}_{2}^{-}$ & tryptophan & tryptophan \\
239.0166 & $\mathrm{C}_{6} \mathrm{H}_{11} \mathrm{~N}_{2} \mathrm{O}_{4} \mathrm{~S}_{2}^{-}$ & cystine & cystine
\end{tabular}

